Article

\title{
Flavonoid Profile of the Genista tridentata L., a Species Used Traditionally to Treat Inflammatory Processes
}

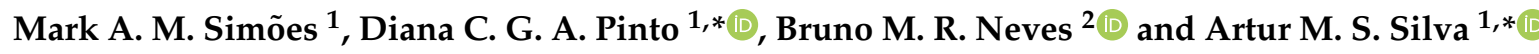 \\ 1 LAQV-REQUIMTE, Department of Chemistry, University of Aveiro, Campus de Santiago, 3810-193 Aveiro, \\ Portugal; mark.simoes@outlook.com \\ 2 Department of Medical Sciences and Institute of Biomedicine-iBiMED, University of Aveiro, \\ 3810-193 Aveiro, Portugal; bruno.neves@ua.pt \\ * Correspondence: diana@ua.pt (D.C.G.A.P.); artur.silva@ua.pt (A.M.S.S.); \\ Tel.: +351-234-401-407 (D.C.G.A.P.); +351-234-370-714 (A.M.S.S.)
}

Academic Editor: Francesco Cacciola

Received: 22 January 2020; Accepted: 11 February 2020; Published: 13 February 2020

check for updates

\begin{abstract}
Ethnopharmacological surveys on Portuguese flora reveal that Genista tridentata L. is a shrub used in traditional medicine for the treatment of various inflammation-related health problems, although scientific support of its benefits is still necessary. In order to establish the anti-inflammatory potential of G. tridentata and support its traditional use, ethanolic extracts of three sections of the plant (root, stem, and leaves) were subjected to in vitro evaluation of anti-inflammatory activity using lipopolysaccharide (LPS)-stimulates macrophages as an inflammation model. Simultaneously, we also aimed to establish the extracts' flavonoids profile. The ethanolic extracts, obtained by Soxhlet extraction, profile of the three sections confirmed their richness in flavonoids, being three prenylated flavonoids isolated and characterized in the root, including a new natural compound, the 3-methoxymundulin. The extracts from the three plant sections showed strong antioxidant activity at the cellular level and significantly inhibit the LPS-triggered NO production by downregulating Nos2 gene transcription and consequently iNOS expression. Additionally, root and stem extracts also decreased the LPS-induced transcription of the pro-inflammatory genes Il1b, Il6, and Ptgs2. Thus, the results support the anti-inflammatory properties attributed to G. tridentate preparations. Relevantly, the roots of the shrub, plant part not used, is an unexplored source of compounds with pharmacological and nutraceutical value.
\end{abstract}

Keywords: Genista tridentata; UHPLC-DAD-ESI/MS ${ }^{n}$ profile; flavonoids; lupinifolin; mundulin; 3-methoxymundulin; anti-inflammatory activity

\section{Introduction}

Genista tridentata L. [1] (Note: Genista tridentata L. is the accepted name of the species and have 13 synonyms and three infraspecific taxa accepted. Among the synonyms Pterospartum tridentatum (L.) Willk. is the most used in the literature and also in the commercially available extracts.) is an endemic shrub of the Iberian Peninsula belonging to the Leguminosae family, locally known as carqueja or carqueija [2]. It grows up spontaneously in the north-eastern region of Portugal, showing coriaceous winged stems, alternate branches, and yellow inflorescences [3]. The flowers are usually collected during spring and used to flavor rice or roasted meat or dried for subsequent preparation of decoctions and infusions. These hot water extracts are believed in folk medicine to present hypoglycemic, anti-hypertensive, and depurative effects being also used for the treatment of numerous inflammation-related health problems $[4,5]$. The water infusions of the shrub aerial 
parts are rich in phenolic compounds and have strong in chemico antioxidant activity [6-8]. In vitro experiments corroborated the antioxidant properties, with human endothelial cells being protected from $\mathrm{H}_{2} \mathrm{O}_{2}$-induced oxidative injury, an effect attributed to isoquercitrin [9]. Flavonols, flavones, and isoflavones are highly abundant in the hidroalcoholic extracts of the plant and confer them a strong antibacterial activity against methicillin-resistant and methicillin-sensitive Staphylococcus aureus [10]. Finally, the polysaccharides present in the dried inflorescence of G. tridentata were also pointed as possible contributors to its health beneficial effects, namely through relevant anti-inflammatory activity [11].

Inflammation is subjacent to multiple health problems, and its mitigation is therefore highly desirable in the treatment of diseases such as rheumatoid arthritis, osteoarthritis, diabetes, atherosclerosis, and Alzheimer's [12,13]. However, available anti-inflammatory drugs have multiple limitations and the development or discovery of novel and safe compounds is of great interest.

The above-mentioned works deal with the aerial parts or flowers and are responsible for the isolation and identification of six isoflavones and three flavone derivatives, from which genistein, prunetin, rutin, and isoquercitrin are due to some already established biological properties [6,7,14-18].

Here, we sought to chemically characterize the ethanolic extracts of the root, stem, and leaves of $G$. tridentat $a$ and to address their antioxidant and anti-inflammatory activities in vitro. Lipopolysaccharidestimulated Raw 264.7 macrophages were used as an inflammation model and the effect of extracts on the production of NO and ROS (reactive oxygen species), as well as on the transcription of Nos2, Il1b, Il6, Ptgs2, and Tnfa was analyzed. Our results support the anti-inflammatory effects attributed in folk medicine to G. tridentata and provide evidence for the possible use of roots and leaves as a source of compounds with valuable pharmacological or nutraceutical properties.

\section{Results and Discussion}

\subsection{Biological Activity of G. Tridentata Ethanolic Extracts}

Some of the health benefits attributed in popular medicine to G. tridentata infusions or decoctions are related to their anti-inflammatory potential [4,5]. However, studies directly addressing these pharmacological effects in G. tridentata preparations are scarce. Thus, the antioxidant and anti-inflammatory activities of ethanolic extracts of the root, stem, and leaves of G. tridentata was evaluated using LPS-stimulated macrophages as an in vitro inflammation model. First, the impact of the extracts on cell viability was addressed in order to select the concentration to be used in subsequent assays. In the range of concentration studied ( 10 to $250 \mathrm{mg} / \mathrm{mL}$ ) the root extract was shown to be less cytotoxic while the extract from leaves caused the higher impact on cell viability (Figure 1). Given the data obtained, the concentration $100 \mu \mathrm{g} / \mathrm{mL}$ was selected, since in all extracts it resulted in no more than $5 \%$ to $10 \%$ cytotoxicity.

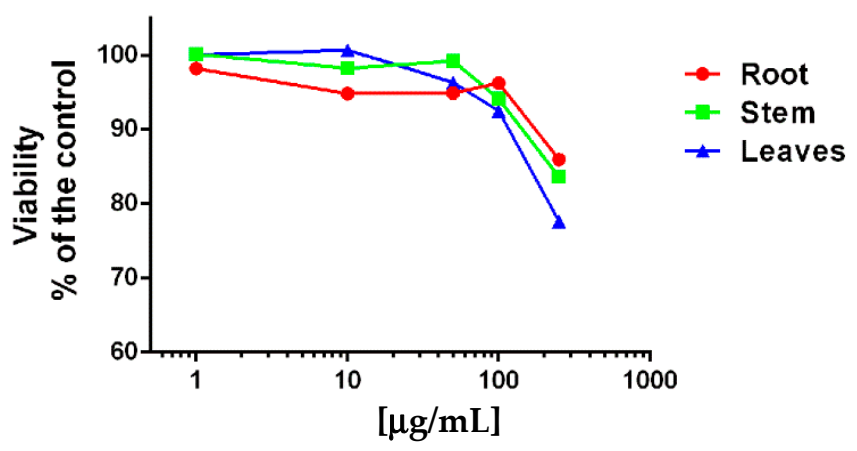

Figure 1. Effect of G. tridentata extracts on Raw 264.7 macrophages viability. Cells were exposed to different concentrations of the extracts, during $24 \mathrm{~h}$ and then viability was assessed by the resazurin assay. Data is presented as percentage of untreated cells (control) and represent the mean from three independent experiments. 
The production of NO by macrophages is critical for the resolution of infectious processes, but its excessive and/or continued production is strongly connected to the development of chronic inflammatory diseases [19]. Therefore, if extracts induce NO per se and if they were able to modulate its LPS-induced production was evaluated (Figure 2a). The incubation of cells with extract from roots or stems did not significantly increase NO levels while extract from leaves caused a slight increase. Looking at the modulation of LPS-triggered NO production it was noticed that all parts of the plant caused a significant decrease (Figure 2a) with this effect as not attributable to direct NO scavenging activity (Figure 2b).

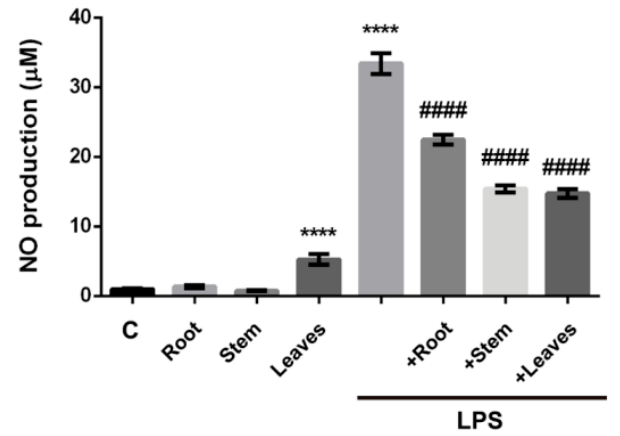

(a)

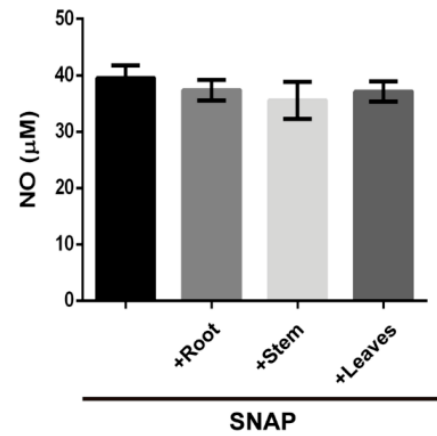

(b)

Figure 2. Evaluation of the capacity of G. tridentata extracts to modulate NO production in Raw 264.7 macrophages. (a) Levels of NO in supernatants from cells stimulated with extracts or with extracts and LPS. (b) Assessment of the potential of NO scavenging activity through the SNAP assays. Data is presented as concentration of $\mathrm{NO}$ and represent mean \pm SD from three independent biological experiments. $\left({ }^{* * * *} p<0.0001\right.$ : control vs. LPS; \#\#\#\# $p<0.0001$ : LPS vs. extracts + LPS). Data is presented as percentage of control (nontreated cells/medium) and represent the mean \pm SD from at least three independent experiments.

The pre-treatment of macrophages with extracts also significantly reduced cellular ROS levels resultant from LPS stimulation (Figure 3). This observation directly relies on the well-established antioxidant properties of flavonoids, metabolites previously found in this species $[6,7,14,20]$, and is in accordance with previous studies demonstrating strong in chemico and in vitro antioxidant activity of G. tridentata extracts $[6,21,22]$. There are evidences in the literature that at least genistein, biochanin A, and daidzein can contribute to the observed effects [23,24]. These compounds have direct ROS scavenging activity and were also shown to increase the expression of superoxide dismutase and glutathione peroxidase, key enzymes in the protection of cell from oxidative damage $[25,26]$.

In order to have a deep understanding on the anti-inflammatory potential of G. tridentata extracts, their capacity to modulate the LPS-triggered transcription of Nos2 and Ptgs2 genes was analyzed by qPCR (Figure 4) and the quantification of the respective coded proteins iNOS and COX-2 was performed by Western blot (Figure 5). The effects on mRNA levels of $I l 1 b$, Il6, and Tnfa were also addressed. All the extracts significantly decreased Nos 2 mRNA levels resulting in a correspondent decrease in protein expression. Therefore, the noticed decrease of LPS-triggered NO production caused by extracts is due to their capacity to limit Nos2 gene transcription, hypothetically by interfering with pro-inflammatory transcription factors such as nuclear factor- $\mathrm{kB}$ (NF- $\mathrm{kB}$ ) and signal transducer and activator of transcription 1 (STAT-1). In fact, the flavonoids genistein, prunetin, biochanin A derivatives, and kampferol have been shown to inhibit NO production by decreasing the activation of NF- $\mathrm{kB}$ signaling pathway $[15,16,27-29]$. 

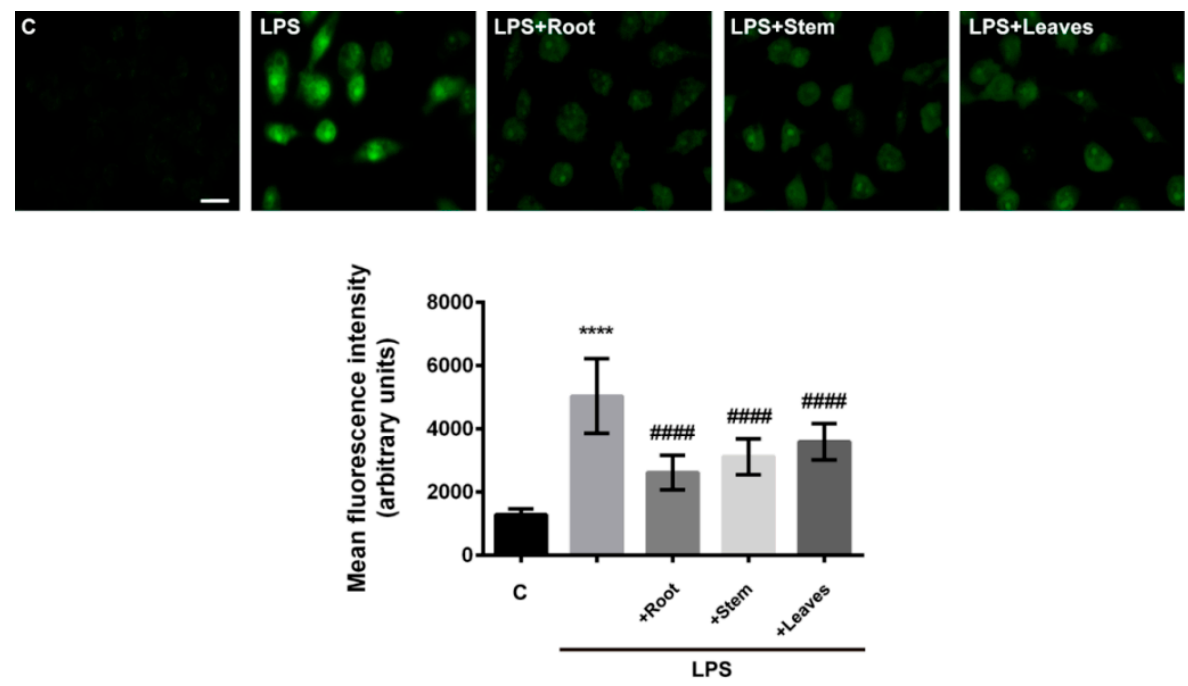

Figure 3. Effects of G. tridentata extracts on the LPS-triggered production of ROS by macrophages. Cells were cultured in the indicated conditions and the ROS production was assessed with $\mathrm{H}_{2}$ DCFDA (green), a ROS-sensitive fluorescent probe. Hoechst (blue) was used to label the nuclei. Images representative of different fields were acquired at a magnification of $63 \times($ scale bar $=20 \mu \mathrm{m})$. Data is presented as mean fluorescence intensity \pm SD from three independent experiments. $(* * * *<0.0001$ : C vs. LPS; \#\#\#\# $p<0.0001$ : LPS vs. extracts + LPS).
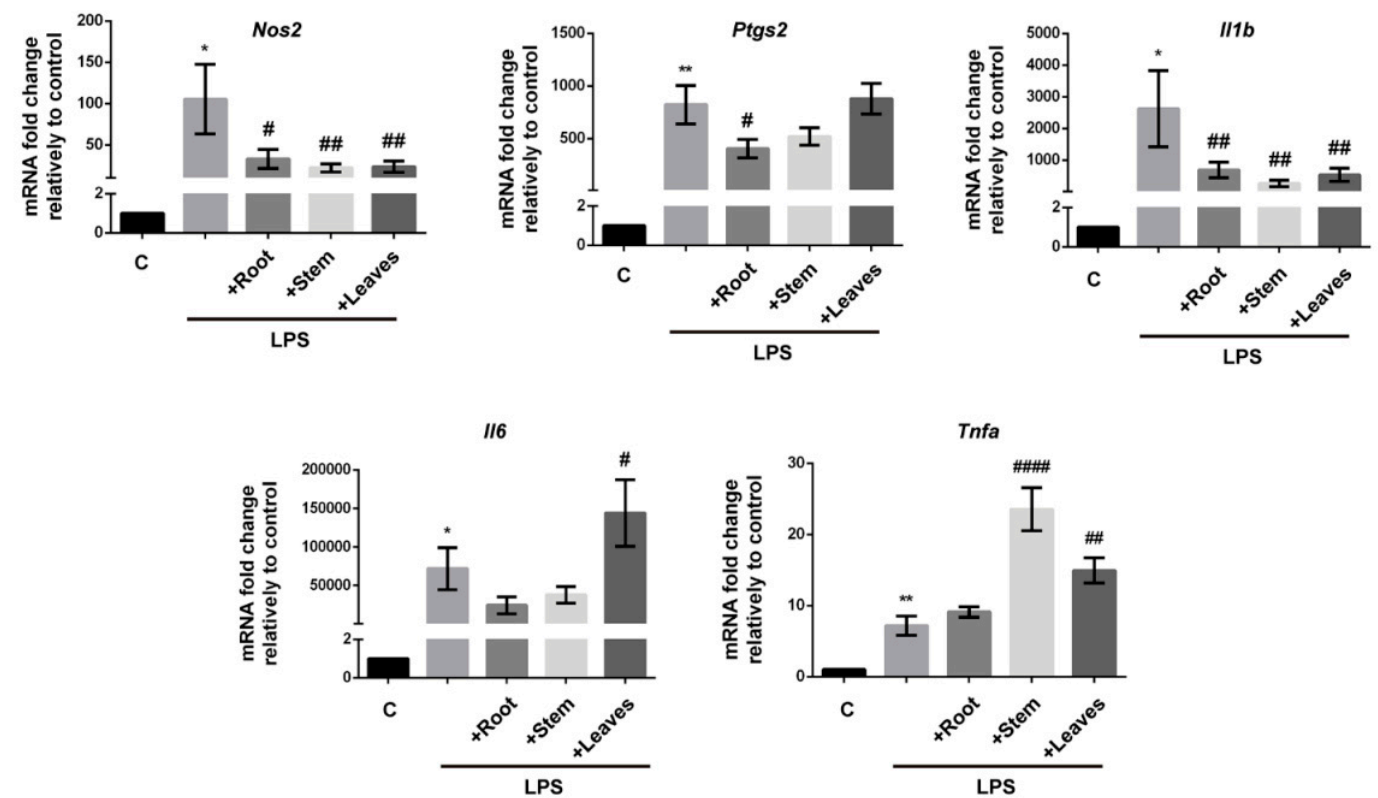

Figure 4. Effects of G. tridentata extracts over LPS-induced transcription of Nos2, Ptgs2, Il1b, Il6, and Tnfa genes. Cells were exposed to extracts from root, stem, and leaves and then stimulated with LPS. After $24 \mathrm{~h}$ the mRNA was extracted and the gene transcription was assessed by q-PCR. Data is presented as mRNA fold change relatively to untreated cells $(C)$ and represent the mean \pm SD from three independent experiments. ( ${ }^{*} p<0.05$; ${ }^{* *} p<0.01$ : C vs. LPS; $\#<0.05$; \#\# $p<0.01$; \#\#\# $p<0.0001$ : LPS vs. extracts + LPS). 
A
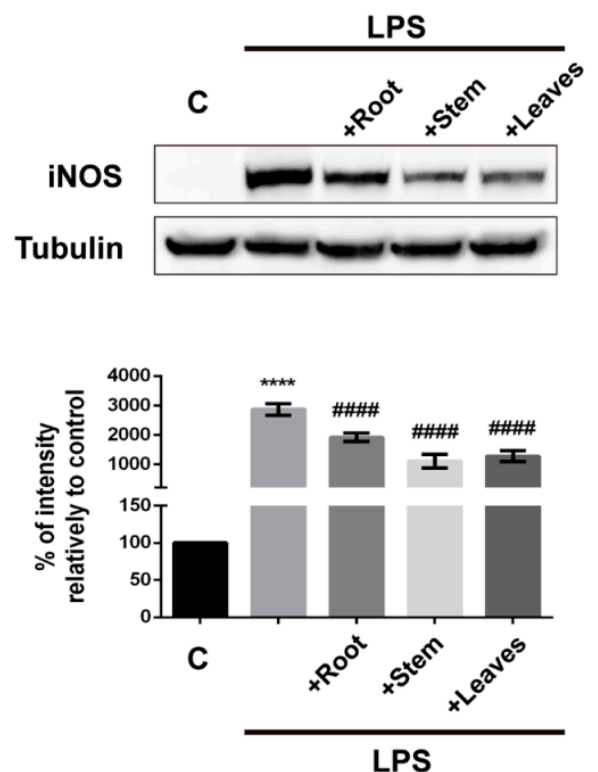

B
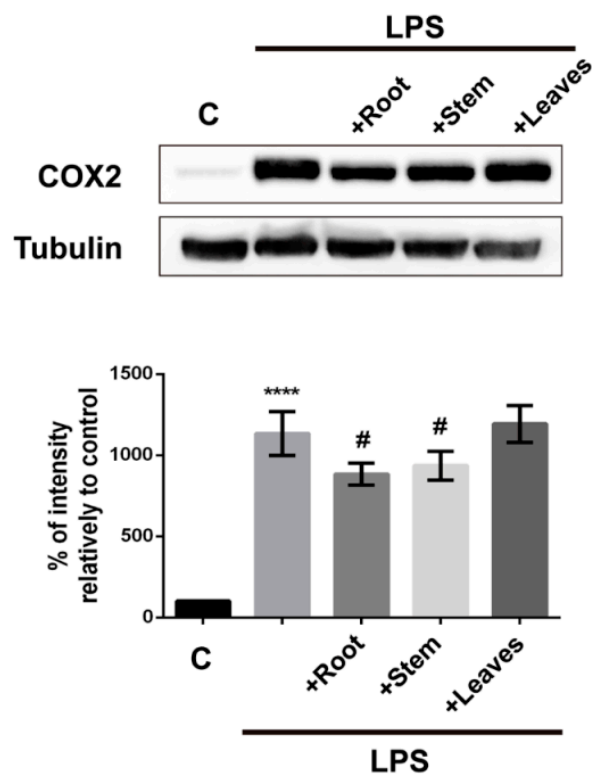

Figure 5. Impact of G. tridentata extracts on the LPS-induced expression of iNOS and COX-2. Cells were exposed to extracts from root, stem, and leaves and then stimulated with LPS. After $24 \mathrm{~h}$ total cell lysates were prepared and iNOS and COX-2 protein levels were assessed by Western blot. The results are expressed as percentage of optical densities relative to untreated cells (control). Equal protein loading was controlled using antibody against $\beta$-tubulin. A representative blot is shown and each value on graphs represents the mean \pm SD of three independent experiments. ${ }^{* * * *} p<0.0001$ : C vs. LPS; \# $p<0.05$, \#\#\#\# $p<0.0001$ : LPS vs. extracts + LPS).

When analyzing the effects over Ptgs2 transcription it was found that significant decreases are only obtained by treatment with extracts from roots (Figure 4). Determination of COX-2 protein levels, the correspondent translation product, followed the same profile, with root extracts being the most effective in limiting the LPS-triggered expression (Figure 5). Given the pivotal role of COX-2 in inflammation we can speculate that, compared to the other plant parts, preparations from G. tridentata roots would have superior anti-inflammatory activity as they would limit prostaglandin production. Again, this effect may be due to the presence of compounds such as genistein and daidzein. Genistein and daidzein are known to exhibit strong inhibitory activity over NF-kB by blocking the degradation of inhibitory $\kappa \mathrm{B}-\alpha(\mathrm{I} \kappa \mathrm{B}-\alpha)$ which results in decreased nuclear translocation of the NF- $\mathrm{kB}$ p50 subunit $[17,30]$. Additionally, these isoflavones were also shown to block STAT-1 phosphorylation, another transcription factor involved in the transcription of Nos2 and Ptgs2 [17,30].

Regarding the effects on the transcription of the other studied inflammatory mediators, the results varied according to the gene and to the plant portion from which the extract was obtained. While LPS-induced transcription of $I l 1 b$ was significantly decreased by all extracts, $I l 6$ and Tnfa genes were either not modulated or their mRNA levels paradoxically increase (Figure 4). The referred effect is particularly evident for Tnfa which was induced by stem and leaf extracts. These results may be explained by the different transcription factors involved in the regulation of each gene. While Il1 $b$ is mainly regulated by NF- $\mathrm{kB}$, a pathway known to be inhibited by several flavonoids, Il6 and Tnfa genes are additionally regulated by other transcription factors. $I l 6$ is regulated by activator protein (AP)- 1 , CCAAT/enhancer binding protein (C/EBP), and cAMP response element-binding protein (CREB) [31] and Tnfa by nuclear factor of activated T-cells (NFAT) along with Sp1 and Ets/Elk [32]. Thus, by our results we can infer that the compounds present in extracts from stem and leaves do not inhibit these transcription factors and in contrast can activate some of them causing the observed synergism with LPS (Figure 4). This reinforces the notion that plant preparations used in folk medicine may contain numerous compounds, some with beneficial properties but others with undesirable characteristics. 


\subsection{Ethanolic Extracts UHPLC-DAD-ESI/MS ${ }^{n}$ Analysis}

The analysis of the phenolic composition of G. tridentata three extracts was established using UHPLC-DAD-ESI/MS ${ }^{n}$ (ultra-high-performance chromatography coupled to photodiode-array detection and electrospray ionization/ion trap mass spectrometry) in the negative ionization mode. For each sample, three independent assays were carried out and similar intensities and nature were observed between the replicates. For each plant part extract the chromatograms were recorded at different wavelengths (Figure 6) but the compounds identification was performed for all samples at the same wavelength. The analysis of the plant parts extracts shows different profiles and that roots extract is less rich in phenolic compounds (Figure 6).
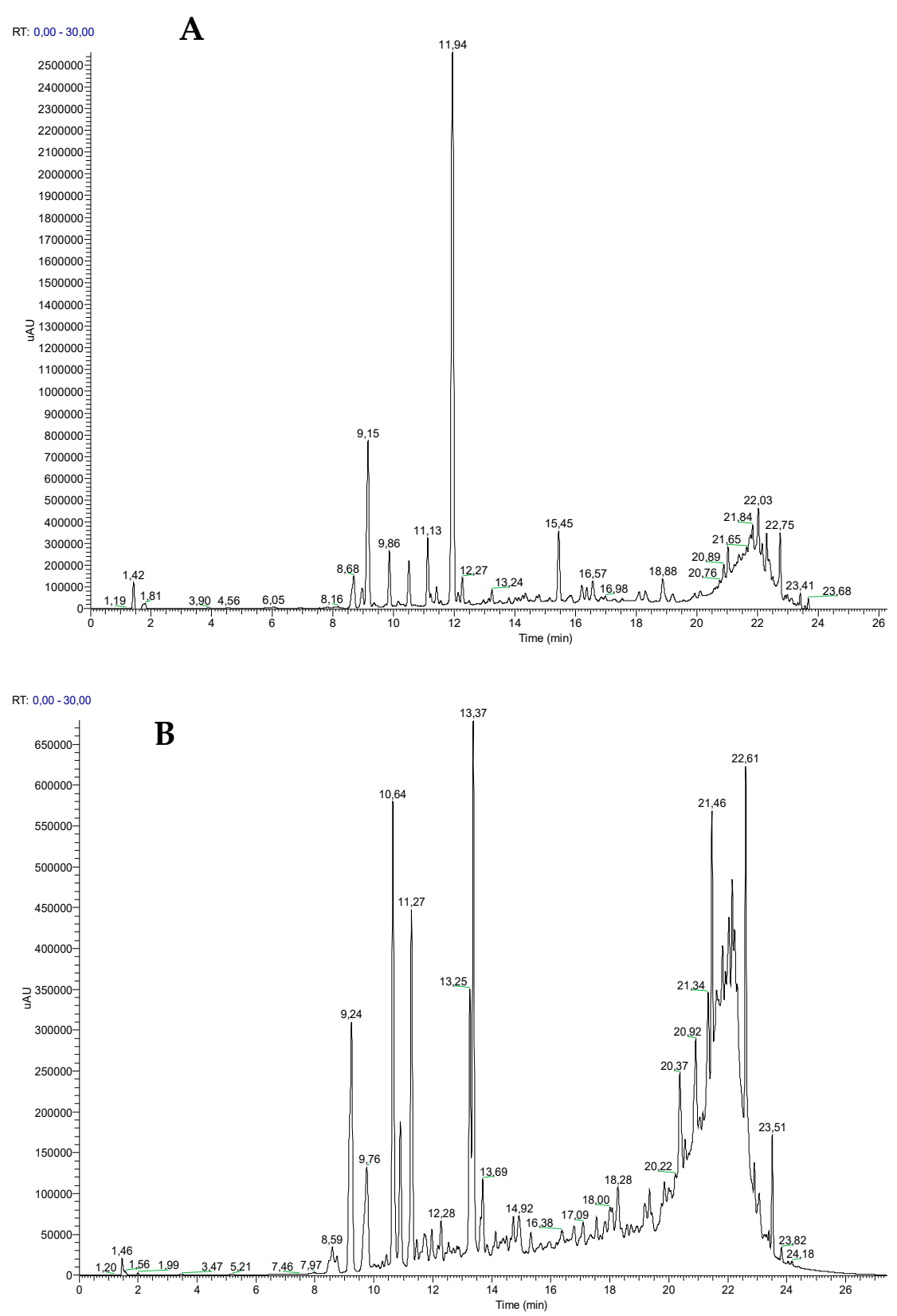

Figure 6. Cont. 


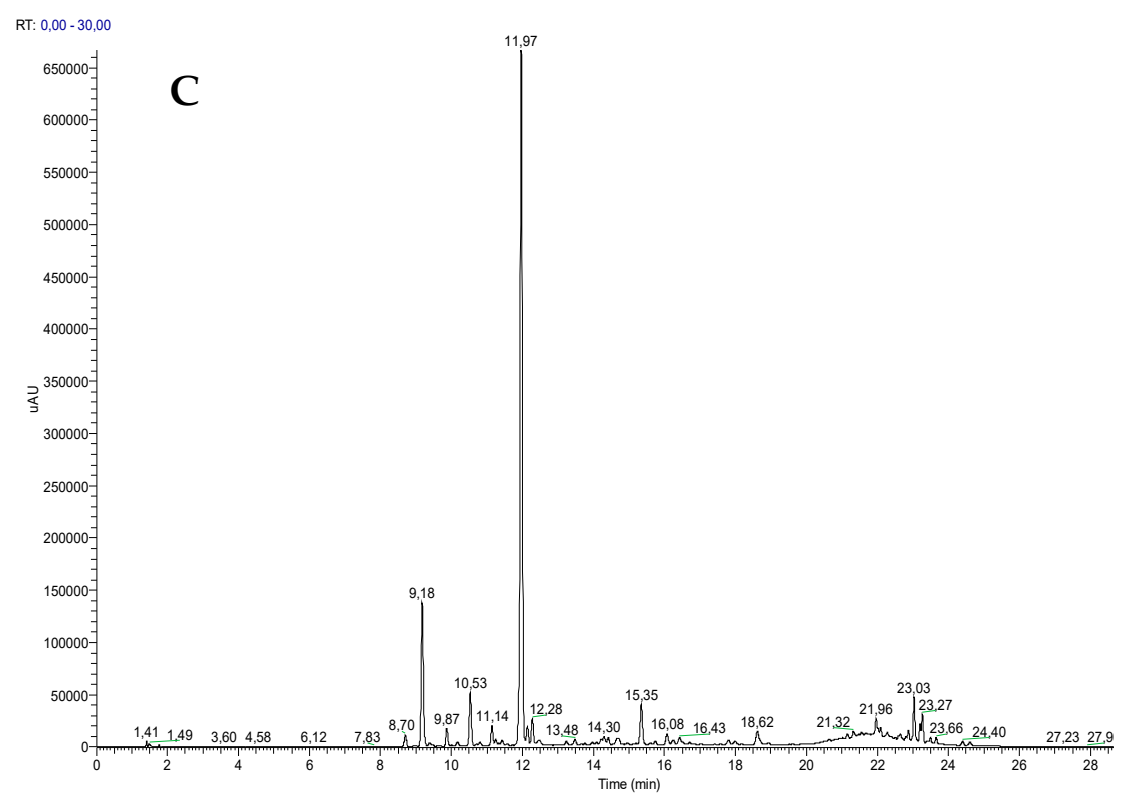

Figure 6. UHPLC chromatograms of G. tridentata extracts. (A) Stems extract (S) recorded at $230 \mathrm{~nm}$; (B) leaves extract $(\mathrm{L})$ recorded at $240 \mathrm{~nm}$; (C) roots extract $(\mathrm{R})$ recorded at $280 \mathrm{~nm}$.

The compounds' characterization was achieved by comparing the deprotonated molecular ion $[\mathrm{M}$ $-\mathrm{H}]^{-}$, although a few formate adducts $\left(\left[\mathrm{M}-\mathrm{H}+\mathrm{CH}_{2} \mathrm{O}_{2}\right]^{-}\right)$were also observed, the fragments in $\mathrm{MS}^{\mathrm{n}}$ and the UV spectra with literature data. Some available standards, both aglycones, and glycosides were injected in the same conditions to confirm some identifications. The analysis of the data resumed in Table 1, permits the confirmation that roots have fewer compounds, nine compounds were identified, whereas leaves and stems have a few extra derivatives. Among the identified compounds (Table 1 ), only genistein aglycone, one of its $C$-glycosides and 5,5'-dihydroxy-3'-methoxyisoflavone 7-O-glucoside (Figure 7) were found in the three analyzed extracts. On the other hand, several compounds were only found in one plant part extract (Table 1). These results also confirmed the richness of this species in isoflavone derivatives, as well as in glycosides. It should be emphasized that in our study the ethanolic extracts were analyzed, whereas in most reported studies water extracts were analyzed. Moreover, in this study the flowers were not included, which is the most studied plant part. These aspects explain the differences between the profiles herein discussed and the previously reported ones, in particular the previously reported richness in quercetin derivatives $[8,20,33]$. 
Table 1. Flavonoid composition of G. tridentata ethanolic extracts. Retention time $\left(t_{R} ;\right.$ min.), wavelengths of maximum absorption in the visible region ( $\lambda_{\text {max }}$; nm), molecular ion $\left([\mathrm{M}-\mathrm{H}]^{-} ; m / z\right)$, mass spectral data $\left(\mathrm{MS}^{n} ; m / z\right)$, roots extract $(\mathrm{R})$, leaves extract $(\mathrm{L})$, and stems extract (S).

\begin{tabular}{|c|c|c|c|c|c|c|c|c|}
\hline $\mathbf{t}_{\mathbf{R}} *$ & $\lambda_{\max }$ & {$[\mathbf{M}-\mathbf{H}]^{-}$} & MS $^{2}$ & MS $^{3}$ & Assigned Identification & $\mathbf{R}$ & $\mathbf{L}$ & $\mathrm{S}$ \\
\hline 8.59 & $\begin{array}{l}198,224,292 \\
\text { sh338 }\end{array}$ & 465 & $\begin{array}{c}447(10)\left[\mathrm{M}-\mathrm{H}-\mathrm{H}_{2} \mathrm{O}^{-}\right. \\
375(9)\left[\mathrm{M}-\mathrm{H}-\mathrm{C}_{3} \mathrm{H}_{6} \mathrm{O}_{3}\right]^{-} \\
345(100)\left[\mathrm{M}-\mathrm{H}-\mathrm{C}_{4} \mathrm{H}_{8} \mathrm{O}_{4}\right]^{-} \\
303(7)[\mathrm{M}-\mathrm{H}-\text { glucose }]^{-}\end{array}$ & $\begin{array}{c}357(9)\left[\mathrm{M}-\mathrm{H}-\mathrm{H}_{2} \mathrm{O}-\mathrm{C}_{3} \mathrm{H}_{6} \mathrm{O}_{3}\right]^{-} \\
327(100)\left[\mathrm{M}-\mathrm{H}-\mathrm{H}_{2} \mathrm{O}-\mathrm{C}_{4} \mathrm{H}_{8} \mathrm{O}_{4}\right]^{-} \\
285(11)\left[\mathrm{M}-\mathrm{H}-\text { glucose }-\mathrm{H}_{2} \mathrm{O}\right]^{-} \\
195(11)\left[\mathrm{M}-\mathrm{H}-\text { glucose }-\mathrm{C}_{6} \mathrm{H}_{4} \mathrm{O}_{2}\right] \\
167(18)\left[\mathrm{M}-\mathrm{H}-\text { glucose }-\mathrm{C}_{7} \mathrm{H}_{4} \mathrm{O}_{3}\right]\end{array}$ & b,c Taxifolin glucoside I & & $\checkmark$ & \\
\hline 8.69 & $192,254, \operatorname{sh} 314$ & 445 & $\begin{array}{c}430(5)\left[\mathrm{M}-\mathrm{H}-\mathrm{CH}_{3}\right]^{-} \\
427(1)\left[\mathrm{M}-\mathrm{H}-\mathrm{H}_{2} \mathrm{O}^{-}\right. \\
355(3)\left[\mathrm{M}-\mathrm{H}-\mathrm{C}_{3} \mathrm{H}_{6} \mathrm{O}_{3}\right]^{-} \\
325(7)\left[\mathrm{M}-\mathrm{H}-\mathrm{C}_{4} \mathrm{H}_{8} \mathrm{O}_{4}\right]^{-} \\
283(100)\left[\mathrm{M}-\mathrm{H}-\text { glucose }^{-}\right. \\
\text {267(2) }[\mathrm{M}-\mathrm{H}-\text { Oglucose }]^{-}\end{array}$ & $\begin{array}{c}\text { 268(100) }\left[\mathrm{M}-\mathrm{H}-\mathrm{CH}_{3}-\text { glucose }\right]^{-} \\
255(2)[\mathrm{M}-\mathrm{H}-\text { glucose }-\mathrm{CO}]^{-}\end{array}$ & ${ }^{\mathrm{c}}$ Sissotrin & & & $\checkmark$ \\
\hline 8.74 & $\begin{array}{l}198,229,291 \\
\quad \operatorname{sh} 335\end{array}$ & 465 & $\begin{array}{c}447(10)\left[\mathrm{M}-\mathrm{H}-\mathrm{H}_{2} \mathrm{O}^{-}\right. \\
375(10)\left[\mathrm{M}-\mathrm{H}-\mathrm{C}_{3} \mathrm{H}_{6} \mathrm{O}_{3}\right]^{-} \\
345(100)\left[\mathrm{M}-\mathrm{H}-\mathrm{C}_{4} \mathrm{H}_{8} \mathrm{O}_{4}\right]^{-} \\
\text {303(7) }[\mathrm{M}-\mathrm{H}-\text { glucose }]^{-}\end{array}$ & $\begin{array}{c}\text { 357(9) }\left[\mathrm{M}-\mathrm{H}-\mathrm{H}_{2} \mathrm{O}-\mathrm{C}_{3} \mathrm{H}_{6} \mathrm{O}_{3}\right]^{-} \\
327(100)\left[\mathrm{M}-\mathrm{H}-\mathrm{H}_{2} \mathrm{O}-\mathrm{C}_{4} \mathrm{H}_{8} \mathrm{O}_{4}\right]^{-} \\
\text {285(11) }\left[\mathrm{M}-\mathrm{H}-\text { glucose }-\mathrm{H}_{2} \mathrm{O}\right]^{-} \\
\text {195(11) }\left[\mathrm{M}-\mathrm{H}-\text { glucose }-\mathrm{C}_{6} \mathrm{H}_{4} \mathrm{O}_{2}\right] \\
\text { 167(18) }\left[\mathrm{M}-\mathrm{H}-\text { glucose }-\mathrm{C}_{7} \mathrm{H}_{4} \mathrm{O}_{3}\right]\end{array}$ & b,c Taxifolin glucoside II & & $\checkmark$ & \\
\hline 9.15 & $\begin{array}{c}194,260,231 \\
330\end{array}$ & 593 & $\begin{array}{c}575(10)\left[\mathrm{M}-\mathrm{H}-\mathrm{H}_{2} \mathrm{O}\right]^{-} \\
503(7)\left[\mathrm{M}-\mathrm{H}-\mathrm{C}_{3} \mathrm{H}_{6} \mathrm{O}_{3}\right]^{-} \\
473(100)\left[\mathrm{M}-\mathrm{H}-\mathrm{C}_{4} \mathrm{H}_{8} \mathrm{O}_{4}\right]^{-} \\
431(7)[\mathrm{M}-\mathrm{H}-\text { glucose }]^{-} \\
415(2)[\mathrm{M}-\mathrm{H}-\text { Oglucose }]^{-} \\
\text {269(7) }[\mathrm{M}-\mathrm{H}-\text { 2glucose }]^{-}\end{array}$ & $\begin{array}{c}311(7)\left[\mathrm{M}-\mathrm{H}-\text { glucose }-\mathrm{C}_{4} \mathrm{H}_{8} \mathrm{O}_{4}\right]^{-} \\
325(2)\left[\mathrm{M}-\mathrm{H}-\text { Oglucose }-\mathrm{C}_{3} \mathrm{H}_{6} \mathrm{O}_{3}\right]^{-} \\
253(2)[\mathrm{M}-\mathrm{H}-\text { Oglucose }- \text { glucose }]^{-}\end{array}$ & $\begin{array}{l}{ }^{c} \text { Genistein } O \text { - and } \\
\text { C-glucoside }\end{array}$ & $\checkmark$ & & $\checkmark$ \\
\hline 9.24 & $\begin{array}{l}199,217,290 \\
\text { sh336 }\end{array}$ & 465 & $\begin{array}{c}447(80)\left[\mathrm{M}-\mathrm{H}-\mathrm{H}_{2} \mathrm{O}\right]^{-} \\
375(15)\left[\mathrm{M}-\mathrm{H}-\mathrm{C}_{3} \mathrm{H}_{6} \mathrm{O}_{3}\right]^{-} \\
345(70)\left[\mathrm{M}-\mathrm{H}-\mathrm{C}_{4} \mathrm{H}_{8} \mathrm{O}_{4}\right]^{-} \\
303(30)[\mathrm{M}-\mathrm{H}-\text { glucose }]^{-} \\
\text {285(100) }\left[\mathrm{M}-\mathrm{H}-\mathrm{H}_{2} \mathrm{O}-\text { glucose }\right]^{-}\end{array}$ & $\begin{array}{l}177(18)\left[\mathrm{M}-\mathrm{H}-\mathrm{H}_{2} \mathrm{O}-\text { glucose }-\mathrm{C}_{6} \mathrm{H}_{4} \mathrm{O}_{2}\right]^{-} \\
151(15)\left[\mathrm{M}-\mathrm{H}-\mathrm{H}_{2} \mathrm{O}-\text { glucose }-\mathrm{C}_{7} \mathrm{H}_{2} \mathrm{O}_{3}\right]^{-}\end{array}$ & b,c Taxifolin glucoside III & $\checkmark$ & $\checkmark$ & \\
\hline 9.76 & 220,265 & 467 & $\begin{array}{c}449(10)\left[\mathrm{M}-\mathrm{H}-\mathrm{H}_{2} \mathrm{O}^{-}\right. \\
345(100)\left[\mathrm{M}-\mathrm{H}-\mathrm{C}_{6} \mathrm{H}_{2} \mathrm{O}_{3}\right]^{-} \\
305(10)\left[\mathrm{M}-\mathrm{H}-\text { glucose }^{-}\right. \\
289(2)[\mathrm{M}-\mathrm{H}-\text { Oglucose }]^{-} \\
\left.\text {275(11) [M- }-\mathrm{H}-\mathrm{CH}_{2}-\text { Oglucose }\right]^{-}\end{array}$ & & $\begin{array}{l}\text { b,c Gallocatechin } \\
\text { 3-O-glucoside }\end{array}$ & & $\checkmark$ & $\checkmark$ \\
\hline
\end{tabular}


Table 1. Cont

\begin{tabular}{|c|c|c|c|c|c|c|c|c|}
\hline$t_{R}^{*}$ & $\lambda_{\max }$ & {$[\mathbf{M}-\mathbf{H}]^{-}$} & MS $^{2}$ & $\mathrm{MS}^{3}$ & Assigned Identification & $\mathbf{R}$ & $\mathbf{L}$ & $\mathrm{S}$ \\
\hline 9.87 & $194,259, \operatorname{sh} 330$ & 639 & $\begin{array}{c}621(8)\left[\mathrm{M}-\mathrm{H}-\mathrm{H}_{2} \mathrm{O}^{-}\right. \\
593(22)\left[\mathrm{M}-\mathrm{H}-\mathrm{CH}_{2} \mathrm{O}_{2}\right]^{-} \\
549(7)\left[\mathrm{M}-\mathrm{H}-\mathrm{C}_{3} \mathrm{H}_{6} \mathrm{O}_{3}\right]^{-} \\
519(83)\left[\mathrm{M}-\mathrm{H}-\mathrm{C}_{4} \mathrm{H}_{8} \mathrm{O}_{4}\right]^{-} \\
477(100)\left[\mathrm{M}-\mathrm{H}-\text { glucose }^{-}\right. \\
431(100)\left[\mathrm{M}-\mathrm{H}-\mathrm{CH}_{2} \mathrm{O}_{2}-\text { glucose }^{-}\right.\end{array}$ & $\begin{array}{c}\text { 269(100) }\left[\mathrm{M}-\mathrm{H}-\mathrm{CH}_{2} \mathrm{O}_{2}-2 \text { glucose }\right]^{-} \\
549(22)\left[\mathrm{M}-\mathrm{H}-\mathrm{CO}_{2}-\mathrm{CH}_{2} \mathrm{O}_{2}\right]^{-} \\
\text {357(6) }\left[\mathrm{M}-\mathrm{H}-\text { glucose }-\mathrm{C}_{4} \mathrm{H}_{8} \mathrm{O}_{4}\right]^{-} \\
\text {315(7) }[\mathrm{M}-\mathrm{H}-2 \text { glucose }]^{-}\end{array}$ & $\begin{array}{l}{ }^{\mathrm{d}} \text { Formic acid genistein } O- \\
\text { and } C \text {-glucoside adduct }\end{array}$ & $\checkmark$ & & $\checkmark$ \\
\hline 10.49 & $198,261, \operatorname{sh} 336$ & 447 & $\begin{array}{c}429(100)\left[\mathrm{M}-\mathrm{H}-\mathrm{H}_{2} \mathrm{O}\right]^{-} \\
403(100)\left[\mathrm{M}-\mathrm{H}-\mathrm{CO}_{2}\right]^{-} \\
357(3)\left[\mathrm{M}-\mathrm{H}-\mathrm{C}_{3} \mathrm{H}_{6} \mathrm{O}_{3}\right]^{-} \\
327(6)\left[\mathrm{M}-\mathrm{H}-\mathrm{C}_{4} \mathrm{H}_{8} \mathrm{O}_{4}\right]^{-} \\
\text {285(10) [M-H - glucose }]^{-} \\
\text {269(30) }[\mathrm{M}-\mathrm{H}-\text { Oglucose }]^{-}\end{array}$ & $\begin{array}{c}267(23)\left[\mathrm{M}-\mathrm{H}-\text { glucose }-\mathrm{H}_{2} \mathrm{O}\right]^{-} \\
257(7)\left[\mathrm{M}-\mathrm{H}-\text { glucose }-\mathrm{CO}^{-}\right. \\
241(27)\left[\mathrm{M}-\mathrm{H}-\text { glucose }-\mathrm{CO}_{2}\right]^{-} \\
217(3)\left[\mathrm{M}-\mathrm{H}-\text { glucose }-\mathrm{C}_{3} \mathrm{O}_{2}\right]^{-} \\
\quad 177(11)^{0,4} \mathrm{~B}^{-} \\
149(16)\left[\mathrm{M}-\mathrm{H}-\text { glucose }-\mathrm{C}_{7} \mathrm{H}_{4} \mathrm{O}_{3}\right]^{-}\end{array}$ & a,c Astragalin & & & $\checkmark$ \\
\hline 10.64 & 221,265 & 467 & $\begin{array}{c}449(10)\left[\mathrm{M}-\mathrm{H}-\mathrm{H}_{2} \mathrm{O}\right]^{-} \\
345(100)\left[\mathrm{M}-\mathrm{H}-\mathrm{C}_{6} \mathrm{H}_{2} \mathrm{O}_{3}\right]^{-} \\
305(10)\left[\mathrm{M}-\mathrm{H}-\text { glucose }^{-}\right. \\
289(2)[\mathrm{M}-\mathrm{H}-\mathrm{Oglucose}]^{-} \\
275(11)\left[\mathrm{M}-\mathrm{H}-\mathrm{CH}_{2}-\text { Oglucose }^{-}\right.\end{array}$ & $257(23)\left[\mathrm{M}-\mathrm{H}-\mathrm{H}_{2} \mathrm{O}-\mathrm{CH}_{2}-\right.$ Oglucose $^{-}$ & $\begin{array}{l}\text { b,c Epigallocatechin } \\
\text { 3-O-glucoside }\end{array}$ & & $\checkmark$ & $\checkmark$ \\
\hline 10.89 & $\begin{array}{l}199,220,290 \\
\quad \operatorname{sh} 340\end{array}$ & 479 & $\begin{array}{c}389(7)\left[\mathrm{M}-\mathrm{H}-\mathrm{C}_{3} \mathrm{H}_{6} \mathrm{O}_{3}\right]^{-} \\
359(100)\left[\mathrm{M}-\mathrm{H}-\mathrm{C}_{4} \mathrm{H}_{8} \mathrm{O}_{4}\right]^{-}\end{array}$ & $\begin{array}{c}341(36)\left[\mathrm{M}-\mathrm{H}-\mathrm{H}_{2} \mathrm{O}-\mathrm{C}_{3} \mathrm{H}_{6} \mathrm{O}_{3}\right]^{-} \\
331(100)\left[\mathrm{M}-\mathrm{H}-\mathrm{CO}-\mathrm{C}_{3} \mathrm{H}_{6} \mathrm{O}_{3}\right]^{-} \\
315(4)\left[\mathrm{M}-\mathrm{H}-\mathrm{CO}_{2}-\mathrm{C}_{3} \mathrm{H}_{6} \mathrm{O}_{3}\right]^{-} 194(15)^{1,4} \mathrm{~B}^{-}\end{array}$ & a,c Myricetin 6-C-glucoside & & $\checkmark$ & \\
\hline 11.14 & $194,255, \operatorname{sh} 328$ & 491 & $\begin{array}{c}445(100)\left[\mathrm{M}-\mathrm{H}-\mathrm{CH}_{2} \mathrm{O}_{2}\right]^{-} \\
\text {283(24) }\left[\mathrm{M}-\mathrm{H}-\mathrm{CH}_{2} \mathrm{O}_{2}-\text { glucose }\right]^{-}\end{array}$ & 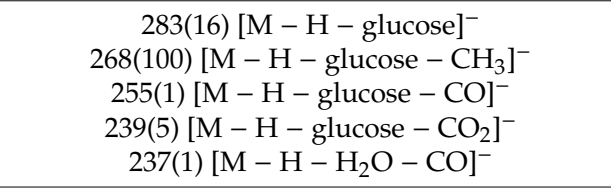 & $\begin{array}{l}\text { d Formic acid biochanin } \\
\text { O-glucoside adduct }\end{array}$ & $\checkmark$ & & $\checkmark$ \\
\hline 13.37 & $\begin{array}{l}206,227,249 \\
271, \operatorname{sh} 328\end{array}$ & 431 & $\begin{array}{c}413(3)\left[\mathrm{M}-\mathrm{H}-\mathrm{H}_{2} \mathrm{O}\right]^{-} \\
341(7)\left[\mathrm{M}-\mathrm{H}-\mathrm{C}_{3} \mathrm{H}_{6} \mathrm{O}_{3}\right]^{-} \\
311(100)\left[\mathrm{M}-\mathrm{H}-\mathrm{C}_{4} \mathrm{H}_{8} \mathrm{O}_{4}\right]^{-} \\
269(13)\left[\mathrm{M}-\mathrm{H}-\text { glucose }^{-}\right.\end{array}$ & $\begin{array}{c}293(15)\left[\mathrm{M}-\mathrm{H}-\mathrm{H}_{2} \mathrm{O}-\mathrm{C}_{4} \mathrm{H}_{8} \mathrm{O}_{4}\right]^{-} \\
\text {282(100) }\left[\mathrm{M}-\mathrm{H}-\mathrm{HCO}-\mathrm{C}_{4} \mathrm{H}_{8} \mathrm{O}_{4}\right]^{-} \\
\text {225(4) }\left[\mathrm{M}-\mathrm{H}-\text { glucose }-\mathrm{CO}_{2}\right]^{-}\end{array}$ & $\begin{array}{l}{ }^{\mathrm{c}} \text { Genistein 6- or } \\
\text { 8-C-glucoside }\end{array}$ & $\checkmark$ & $\checkmark$ & $\checkmark$ \\
\hline 13.69 & $192,262, \operatorname{sh} 332$ & 461 & $\begin{array}{c}446(12)\left[\mathrm{M}-\mathrm{H}-\mathrm{CH}_{3}\right]^{-} \\
443(1)\left[\mathrm{M}-\mathrm{H}-\mathrm{H}_{2} \mathrm{O}\right]^{-} \\
371(7)\left[\mathrm{M}-\mathrm{H}-\mathrm{C}_{3} \mathrm{H}_{6} \mathrm{O}_{3}\right]^{-} \\
341(7)\left[\mathrm{M}-\mathrm{H}-\mathrm{C}_{4} \mathrm{H}_{8} \mathrm{O}_{4}\right]^{-} \\
\text {299(23) }[\mathrm{M}-\mathrm{H}-\text { glucose }]^{-} \\
\text {283(38) }[\mathrm{M}-\mathrm{H}-\text { Oglucose }]^{-}\end{array}$ & $\begin{array}{c}326(3)\left[\mathrm{M}-\mathrm{H}-\mathrm{CH}_{3}-\mathrm{C}_{4} \mathrm{H}_{8} \mathrm{O}_{4}\right]^{-} \\
\text {268(2) }\left[\mathrm{M}-\mathrm{H}-\mathrm{CH}_{3}-\mathrm{Oglucose}\right]^{-} \\
\text {255(4) }[\mathrm{M}-\mathrm{H}-\mathrm{CO}-\text { Oglucose }]^{-}\end{array}$ & $\begin{array}{l}\mathrm{c} \\
\text { 5,5'-Dihydroxy-3'-methoxyis } \\
\text { 7-O-glucoside }\end{array}$ & flatione & & $\checkmark$ \\
\hline
\end{tabular}


Table 1. Cont.

\begin{tabular}{|c|c|c|c|c|c|c|c|c|}
\hline$t_{R}^{*}$ & $\lambda_{\max }$ & {$[\mathbf{M}-\mathbf{H}]^{-}$} & MS $^{2}$ & MS $^{3}$ & Assigned Identification & $\mathbf{R}$ & $\mathbf{L}$ & $S$ \\
\hline 14.73 & $196,260, \operatorname{sh} 334$ & 477 & $\begin{array}{c}459(9)\left[\mathrm{M}-\mathrm{H}-\mathrm{H}_{2} \mathrm{O}^{-}\right. \\
433(6)\left[\mathrm{M}-\mathrm{H}-\mathrm{CO}_{2}\right]^{-} \\
387(7)\left[\mathrm{M}-\mathrm{H}-\mathrm{C}_{3} \mathrm{H}_{6} \mathrm{O}_{3}\right]^{-} \\
357(70)\left[\mathrm{M}-\mathrm{H}-\mathrm{C}_{4} \mathrm{H}_{8} \mathrm{O}_{4}\right]^{-} \\
315(100)[\mathrm{M}-\mathrm{H}-\text { glucose }]^{-} \\
\text {297(10) }\left[\mathrm{M}-\mathrm{H}-\mathrm{H}_{2} \mathrm{O}-\text { glucose }^{-}\right.\end{array}$ & $\begin{array}{c}297(75)\left[\mathrm{M}-\mathrm{H}-\mathrm{H}_{2} \mathrm{O}-\text { glucose }^{-}\right. \\
\text {282(100) }\left[\mathrm{M}-\mathrm{H}-\mathrm{H}_{2} \mathrm{O}-\text { glucose }-\mathrm{CH}_{3}\right]^{-} \\
269(13)\left[\mathrm{M}-\mathrm{H}-\mathrm{H}_{2} \mathrm{O}-\text { glucose }-\mathrm{CO}\right]^{-}\end{array}$ & $\begin{array}{l}\text { a,c Isorhamnetin } \\
\text { 3-O-glucoside }\end{array}$ & & $\checkmark$ & \\
\hline 15.35 & $255, \operatorname{sh} 318$ & 283 & $\begin{array}{c}268(100)\left[\mathrm{M}-\mathrm{H}-\mathrm{CH}_{3}\right]^{-} \\
255(2)[\mathrm{M}-\mathrm{H}-\mathrm{CO}]^{-} \\
239(4)\left[\mathrm{M}-\mathrm{H}-\mathrm{CO}_{2}\right]^{-} \\
165(1){ }^{1,3} \mathrm{~A}^{-}\end{array}$ & 196(1) $\left[\mathrm{M}-\mathrm{H}-\mathrm{CH}_{3}-\mathrm{CO}_{2}-\mathrm{CO}\right]^{-}$ & ${ }^{\mathrm{c}}$ Prunetin & $\checkmark$ & & $\checkmark$ \\
\hline 16.08 & $249, \operatorname{sh} 295,301$ & 253 & $\begin{array}{c}225(70)\left[\mathrm{M}-\mathrm{H}-\mathrm{CO}^{-}\right. \\
211(3)\left[\mathrm{M}-\mathrm{H}-\mathrm{C}_{2} \mathrm{H}_{2} \mathrm{O}\right]^{-} \\
209(7)\left[\mathrm{M}-\mathrm{H}-\mathrm{CO}_{2}\right]^{-} \\
135(2)^{1,3} \mathrm{~A}^{-} \\
117(1)^{1,3} \mathrm{~B}^{-}\end{array}$ & 197(7) $\left[\mathrm{M}-\mathrm{H}-\mathrm{CO}_{2}-\mathrm{CO}^{-}\right.$ & ${ }^{\mathrm{c}}$ Daidzein & $\checkmark$ & & \\
\hline 16.57 & $198,261, \operatorname{sh} 294$ & 285 & $\begin{array}{c}267(4)\left[\mathrm{M}-\mathrm{H}-\mathrm{H}_{2} \mathrm{O}^{-}\right. \\
257(51)\left[\mathrm{M}-\mathrm{H}-\mathrm{CO}^{-}\right. \\
241(27)\left[\mathrm{M}-\mathrm{H}-\mathrm{CO}_{2}\right]^{-} \\
217(53)\left[\mathrm{M}-\mathrm{H}-\mathrm{C}_{3} \mathrm{O}_{2}\right]^{-} \\
177(7){ }^{0,4} \mathrm{~B}^{-} \\
149(11)\left[\mathrm{M}-\mathrm{H}-\mathrm{C}_{7} \mathrm{H}_{4} \mathrm{O}_{3}\right]^{-}\end{array}$ & $\begin{array}{c}229(4)[\mathrm{M}-\mathrm{H}-2 \mathrm{CO}]^{-} \\
199(72)\left[\mathrm{M}-\mathrm{H}-\mathrm{H}_{2} \mathrm{O}-\mathrm{C}_{3} \mathrm{O}_{2}\right]^{-}\end{array}$ & a,c Kaempferol & & & $\checkmark$ \\
\hline 17.09 & $194,255, \operatorname{sh} 320$ & 283 & $\begin{array}{c}269(3)\left[\mathrm{M}-\mathrm{CH}_{3}\right]^{-} \\
268(55)\left[\mathrm{M}-\mathrm{H}-\mathrm{CH}_{3}\right]^{-} \\
265(10)\left[\mathrm{M}-\mathrm{H}-\mathrm{H}_{2} \mathrm{O}\right]^{-} \\
255(91)[\mathrm{M}-\mathrm{H}-\mathrm{CO}]^{-} \\
151(1)^{1,3} \mathrm{~A}^{-} \\
132(1)^{1,3} \mathrm{~B}^{-} \\
149(4)^{0,3} \mathrm{~B}^{-}\end{array}$ & $\begin{array}{c}237(4)\left[\mathrm{M}-\mathrm{H}-\mathrm{H}_{2} \mathrm{O}-\mathrm{CO}\right]^{-} \\
212(5)\left[\mathrm{M}-\mathrm{H}-\mathrm{CH}_{3}-2 \mathrm{CO}^{-}\right. \\
\text {181(3) }\left[\mathrm{M}-\mathrm{CH}_{3}-2 \mathrm{CO}_{2}\right]^{-}\end{array}$ & ${ }^{c}$ Biochanin A & & $\checkmark$ & \\
\hline 18.88 & $195,261, \operatorname{sh} 332$ & 269 & $\begin{array}{c}251(12)\left[\mathrm{M}-\mathrm{H}-\mathrm{H}_{2} \mathrm{O}\right]^{-} \\
241(86)\left[\mathrm{M}-\mathrm{H}-\mathrm{CO}^{-}\right. \\
225(97)\left[\mathrm{M}-\mathrm{H}-\mathrm{CO}_{2}\right]^{-} \\
201(10)\left[\mathrm{M}-\mathrm{H}-\mathrm{C}_{3} \mathrm{O}_{2}\right]^{-} \\
151(10)^{1,3} \mathrm{~A}^{-} \\
117(4){ }^{1,3} \mathrm{~B}^{-}\end{array}$ & $\begin{array}{c}\text { 197(17) }\left[\mathrm{M}-\mathrm{H}-\mathrm{CO}_{2}-\mathrm{CO}^{-}\right. \\
181(3)\left[\mathrm{M}-\mathrm{H}-2 \mathrm{CO}_{2}\right]^{-}\end{array}$ & ${ }^{c}$ Genistein & $\checkmark$ & $\checkmark$ & $\checkmark$ \\
\hline
\end{tabular}


Table 1. Cont.

\begin{tabular}{|c|c|c|c|c|c|c|c|c|}
\hline$t_{\mathbf{R}}^{*}$ & $\lambda_{\max }$ & {$[\mathbf{M}-\mathbf{H}]^{-}$} & MS $^{2}$ & MS $^{3}$ & Assigned Identification & $\mathbf{R}$ & $\mathbf{L}$ & $\mathrm{S}$ \\
\hline 21.03 & $194,260, \operatorname{sh} 320$ & 297 & $\begin{array}{c}282(10)\left[\mathrm{M}-\mathrm{H}-\mathrm{CH}_{3}\right]^{-} \\
279(4)\left[\mathrm{M}-\mathrm{H}-\mathrm{H}_{2} \mathrm{O}\right]^{-} \\
269(6)\left[\mathrm{M}-\mathrm{H}-\mathrm{CO}^{-}\right. \\
253(3)\left[\mathrm{M}-\mathrm{H}-\mathrm{CO}_{2}\right]^{-}\end{array}$ & $\begin{array}{c}241(3)\left[\mathrm{M}-\mathrm{H}-2 \mathrm{CO}^{-}\right. \\
225(5)\left[\mathrm{M}-\mathrm{H}-\mathrm{CO}-\mathrm{CO}_{2}\right]^{-}\end{array}$ & $\frac{c}{5-H y d r o x y-4^{\prime}, 7-d i m e t h o x y i}$ & lavone & & $\checkmark$ \\
\hline
\end{tabular}

${ }^{*}$ Average of three independent injections at the same wavelength $(280 \mathrm{~nm})^{\mathrm{a}}$ Identified based on $\mathrm{MS}^{\mathrm{n}}$ and UV data, and comparison with a standard; ${ }^{\mathrm{b}}$ identified based on MS ${ }^{\mathrm{n}}$ and $\mathrm{UV}$ data, and comparison with a similar standard; ${ }^{c}$ identified based on $\mathrm{MS}^{\mathrm{n}}$ and UV data, and comparison with other data from reference sources; ${ }^{\mathrm{d}}$ tentatively identified based on MS ${ }^{\mathrm{n}}$ and UV data, and other literature evidence. 


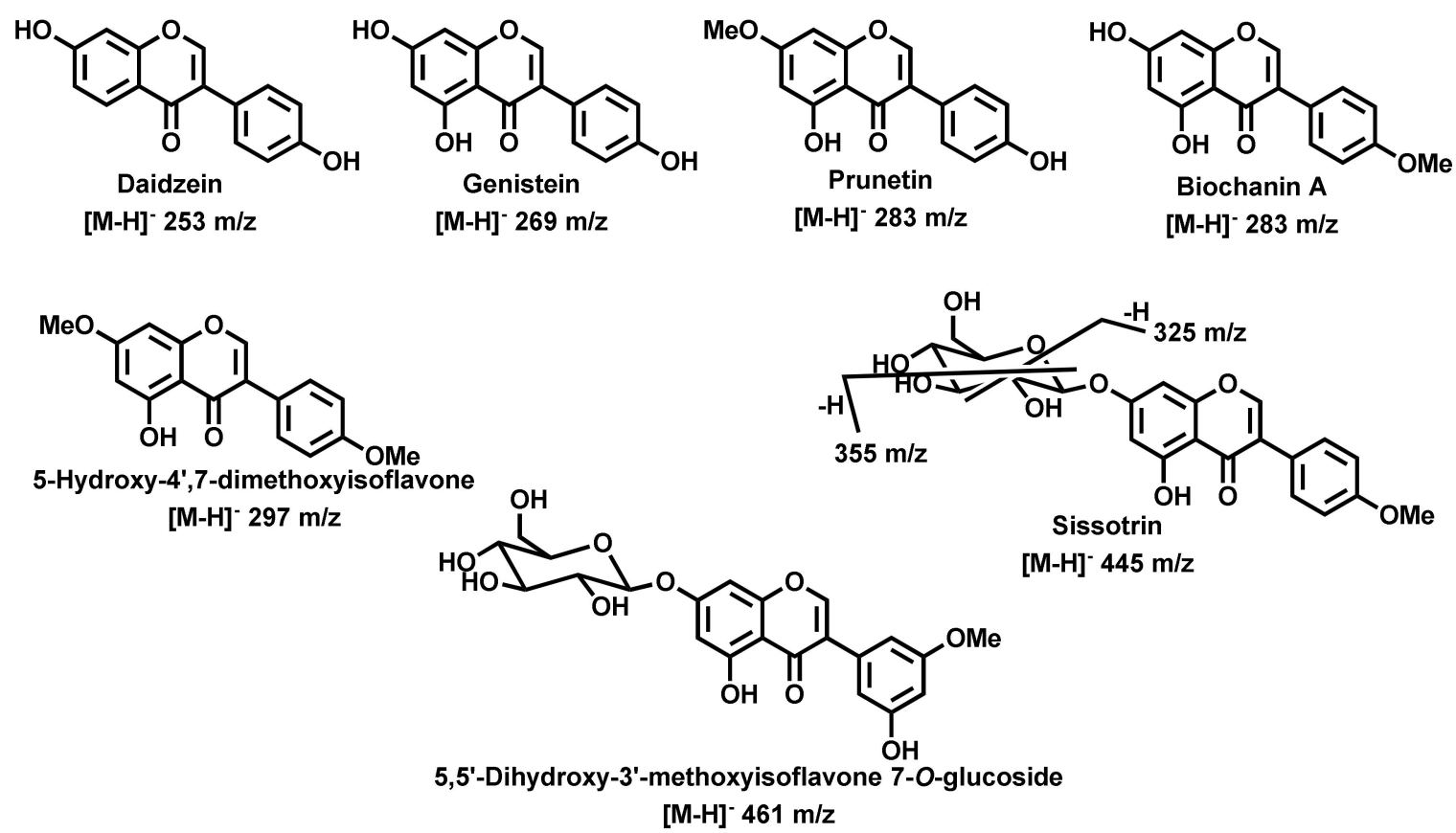

Figure 7. Isoflavonoid derivatives identified in G. tridentata extracts.

\subsubsection{Isoflavonoid Derivatives}

Daidzein (Figure 7), only found in roots extract, exhibited $[\mathrm{M}-\mathrm{H}]^{-}$at $m / z 253$ and presented a fragment pattern consistent with literature available data [34], from which fragments at $m / z 225$ and 209 , due to losses of $\mathrm{CO}$ and $\mathrm{CO}_{2}$, respectively, can be highlighted. Moreover, the isoflavones characteristic cleavages [34], that is fragments at $m / z 135$ and 117 , respectively $1,3 \mathrm{~A}^{-}$and $1,3 \mathrm{~B}^{-}$, can also be observed. Another important isoflavone found in all extracts was genistein (Figure 7). It exhibited [M - H] $]^{-}$ at $m / z 269$ and presented a fragment pattern (Table 1) consistent with literature available data [34], that allowed its assignment. The other isoflavone aglycones found were well known methylated derivatives of genistein (Table 1 ) that exhibited the expected molecular ions $[\mathrm{M}-\mathrm{H}]^{-}$and from which 5-hydroxy-4', 7-dimethoxyisoflavone (Figure 7), only found in stems extract and identified by other authors as methylbiochanin A/methylprunetin [8,20]. Biochanin A and prunetin (Figure 7) isomers can be easily distinguished by the fragment ions at $m / z 165$ and 151, respectively in prunetin and biochanin $\mathrm{A}$, due to the $1,3 \mathrm{~A}^{-}$fragments. In the case of prunetin this fragment is consistent with the presence of a 7-OMe group (Figure 7).

The isoflavone glycosides found were also the expected ones, mainly genistein derivatives (Figure 7). Sissotrin, only found in stems extract, whose occurrence was previously reported in this species [8], presented the characteristic fragments at $m / z 355,325$, and 283, due to losses of 90, 120, and $162 \mathrm{Da}$, characteristic when a glucoside moiety is present (Figure 7). Moreover, a fragment at m/z 267, indicating the loss of $178 \mathrm{Da}$, confirms the 7-O-glucose moiety. 5,5'-Dihydroxy-3'-methoxyisoflavone 7-O-glucoside (Figure 7), exhibited the expected molecular ion $[\mathrm{M}-\mathrm{H}]^{-}$and the observed fragmentation is similar to the previous reported mechanism [9].

Finally, the genistein glycosides presented the expected molecular ions $[\mathrm{M}-\mathrm{H}]^{-}$consistent with the presence of two or one glucoside moieties (Table 1). The derivative eluted at 9.15 min was assigned to a genistein diglucoside derivative, mainly due to its fragmentation consistency with losses of two glucose units. Furthermore, the loss of both 178 and $162 \mathrm{Da}$, corresponding, respectively, to O-glucose and glucose moieties allowed the assignment to a genistein $O$ - and $C$-glucoside. However, in this study attempts to confirm the glucose moieties localization were not performed and we only know that it is a genistein $\mathrm{C}, \mathrm{O}$-glucoside derivative. A similar identification could be performed with the derivative eluted at $13.37 \mathrm{~min}$, the occurrence of genistein 8-C-glucoside was previously reported [8,33], however 
we did not inject standards to confirm the localization of the glucose moiety. Two other isoflavone derivatives identified should be mentioned, the compounds eluted at 9.87 and $11.14 \mathrm{~min}$, which were assigned to formic acid adducts. These adducts are common when a percentage of formic acid is used in the eluent, which was our case.

\subsubsection{Flavonol and Flavanonol Derivatives}

Kaempferol (Figure 8) was the only aglycone, from this type of flavonoids, found, all the others were detected in their glycoside form (Table 1). Interesting is the fact that both kaempferol and astragalin, the kaempferol 3-O-glucoside, were detected in stems extract. Apparently, this is the first report on their occurrence in this species, however their natural occurrence is well reported and our data are in accordance with the previously reported data, which were also used to establish our assignment [35-38].<smiles>O=c1c(O)c(-c2ccc(O)cc2)oc2cc(O)cc(O)c12</smiles>

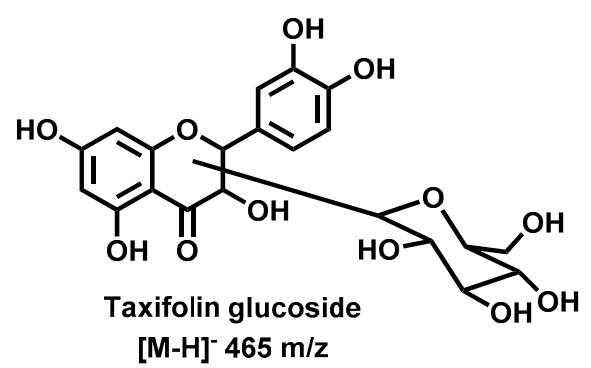<smiles>CC(C)C(O)C(O)C(O)C(O)C(O)Oc1c(-c2ccc(O)cc2)oc2cc(O)cc(O)c2c1=O</smiles>

[M-H]- $447 \mathrm{~m} / \mathrm{z}$<smiles>COc1cc(-c2oc3cc(O)cc(O)c3c(=O)c2OC2OC(O)C(O)C(O)C(O)C2O)ccc1O</smiles>

Isorhamnetin 3-O-glucoside $[\mathrm{M}-\mathrm{H}]^{-} 477 \mathrm{~m} / \mathrm{z}$

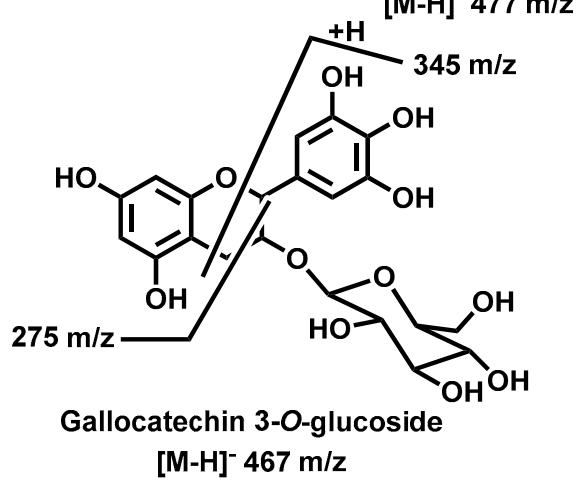

Figure 8. Other polyphenolic derivatives identified in G. tridentata extracts.

Myricetin 6-C-glucoside (Figure 8), assigned to the compound eluted at $10.89 \mathrm{~min}$, was previously found in the G. tridentata extract [14,33], as well as isorhamnetin 3-O-glucoside (Figure 8), assigned to the compound eluted at $10.89 \mathrm{~min}$. [39]. In both cases our data are in accordance with the previous reported $[14,33,39,40]$. The presence of taxifolin glucosides in this species, in particular taxifolin 6-C-glucoside [8,33], was also reported. In this study taxifolin glucosides were found in all extracts, the compounds eluted at $8.59,8.74$, and $9.24 \mathrm{~min}$ (Table 1). Not only is our data similar to the previous reported one but also the occurrence of isomeric taxifolin glucosides is common [41], which explains our results. Although we detected three taxifolin glucosides we could not assign the exact position of the glucose moiety.

\subsubsection{Flavan-3-ol Derivatives}

Compounds eluted at 9.76 and $10.64 \mathrm{~min}$, were assigned respectively to gallocatechin 3-O-glucoside and epigallocatechin 3-O-glucoside (Figure 8) based on previous reported data concerning the flavan-3-ols fragmentation [41,42]. The losses of 162 and $178 \mathrm{Da}$, indicating the presence of an O-glucose unit, and the fragment ions $\mathrm{m} / \mathrm{z} 345$ and 275, common in flavan-3-ols (Figure 8), are the main characteristic of these compounds. 


\subsection{Characterization of the Isolated Metabolites}

Due to the above-mentioned biological potential of the roots extract and the fact that this plant part was always neglected, indeed the plant aerial parts are the ones used in the medicines preparation [4], a phytochemical study was performed. Three prenylated flavonoids (Figure 9), lupinifolin, mundulin, and 3-methoxymundulin, a new natural compound, were isolated. First the extract was submitted to a liquid-solid extraction and a series of chromatographic techniques that allowed the purification of the mentioned flavanone derivatives. Comparison of the NMR spectroscopic data with the previous reported one led to the identification of lupinifolin [43] and mundulin [44], compounds found for the first time in this species. As far as we could find, 3-methoxymundulin (Figure 9) is a new natural derivative and consequently a careful characterization was performed. The mass spectrum showed peaks at $m / z 421,443$, and 459 , due to ions $[\mathrm{M}+\mathrm{H}]^{+},[\mathrm{M}+\mathrm{Na}]^{+}$, and $[\mathrm{M}+\mathrm{K}]^{+}$, respectively. The HRMS-ESI analysis showed a peak at $m / z 421.2021$ assigned to the molecular formula $\mathrm{C}_{26} \mathrm{H}_{29} \mathrm{O}_{5}$. The ${ }^{1} \mathrm{H}-\mathrm{NMR}$ spectrum indicated signals for a hydrogen-bonded hydroxyl group at $\delta_{\mathrm{H}} 11.99 \mathrm{ppm}(1 \mathrm{H}$, $\mathrm{s}, 5-\mathrm{OH})$, a monosubstituted aromatic ring at $\delta_{\mathrm{H}} 7.44-7.48 \mathrm{ppm}(5 \mathrm{H}, \mathrm{m})$, two methyl groups at $\delta_{\mathrm{H}} 1.43$ $\left(3 \mathrm{H}, \mathrm{s}, 2^{\prime \prime \prime}-\mathrm{CH}_{3}\right)$, and $1.45 \mathrm{ppm}\left(3 \mathrm{H}, \mathrm{s}, 2^{\prime \prime \prime}-\mathrm{CH}_{3}\right)$, a methoxyl group at $\delta_{\mathrm{H}} 3.39 \mathrm{ppm}\left(3 \mathrm{H}, \mathrm{s}, 3-\mathrm{OCH}_{3}\right)$ and a vinylic system at $\delta_{\mathrm{H}} 5.51 \mathrm{ppm}\left(1 \mathrm{H}, \mathrm{d}, J=9.2 \mathrm{~Hz}, \mathrm{H}-3^{\prime \prime \prime}\right)$ and at $\delta_{\mathrm{H}} 6.63 \mathrm{ppm}(1 \mathrm{H}, \mathrm{d}, J=9.2 \mathrm{~Hz}$, $\left.\mathrm{H}-4^{\prime \prime \prime}\right)$. The prenyl unit was identified from the resonance of one olefinic proton at $\delta_{\mathrm{H}} 5.12 \mathrm{ppm}(1 \mathrm{H}, \mathrm{t}$, $\left.J=6.0 \mathrm{~Hz}, \mathrm{H}-2^{\prime \prime}\right)$, two methylene protons at $\delta_{\mathrm{H}} 3.19 \mathrm{ppm}\left(2 \mathrm{H}, \mathrm{d}, J=6.0 \mathrm{~Hz}, \mathrm{H}-1^{\prime \prime}\right)$ and two methyl groups at $\delta_{\mathrm{H}} 1.64 \mathrm{ppm}\left(6 \mathrm{H}, \mathrm{s}, 4^{\prime \prime}-\right.$, and $\left.5^{\prime \prime}-\mathrm{CH}_{3}\right)$. Finally, the protons of the flavanone heterocyclic ring $\mathrm{H}-2$ and $\mathrm{H}-3$ resonate at $\delta_{\mathrm{H}} 5.22 \mathrm{ppm}(1 \mathrm{H}, \mathrm{d}, J=10.0 \mathrm{~Hz}, \mathrm{H}-2)$ and $\delta_{\mathrm{H}} 4.04 \mathrm{ppm}(1 \mathrm{H}, \mathrm{t}, J=10.0 \mathrm{~Hz}$, $\mathrm{H}-3)$, respectively. COSY correlations confirm olefinic protons assignments, as well as the assignments of both protons H-2 and H-3. The HMBC cross-peaks (Figure 9) joined with HSQC correlations allowed the carbons assignment, as well as the confirmation of the structure depicted in Figure 9.<smiles>CC(C)=CCc1c2c(c(O)c3c1O[C@H](c1ccccc1)CC3=O)C=CC(C)(C)O2</smiles>

Mundulin
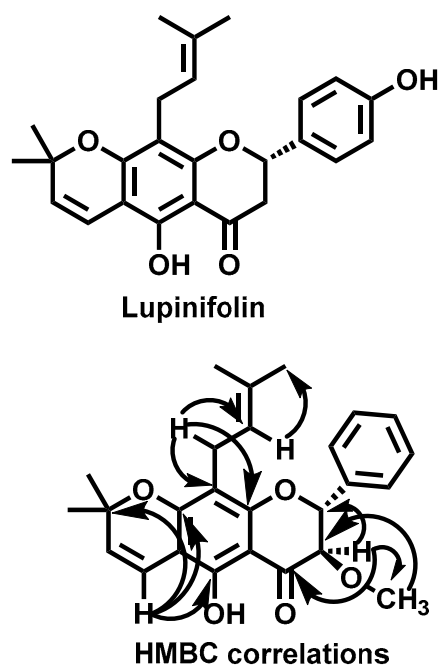

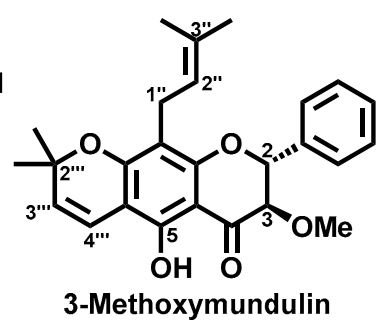

3-Methoxymundulin

Figure 9. Prenylated flavonoids isolated from G. tridentata roots extract.

Previous reports have revealed that both lupinifolin and mundulin present interesting biological activities, such as antimicrobial [45], antitumor [46], and ability to inhibit the $\mathrm{H}^{+}, \mathrm{K}^{+}$-ATPase enzyme [47]. Therefore, the isolation of this flavanone derivatives point out to the G. tridentata roots biological potential.

\section{Materials and Methods}

\subsection{General Experimental Procedures}

Solvents were purchased from Panreac and Acros Organics (Porto Salvo, Portugal) and were of HPLC purity, analytical grade, or bi-distilled commercial solvents. Chromatographic purifications 
were performed using silica gel 60 (Merck Kieselgel, 70-230 mesh) and Merck silica gel $60 \mathrm{GF}_{254}$ (Oeiras, Portugal). Spots were detected in the TLC plates under UV light at $\lambda 254$ and $366 \mathrm{~nm}$. The phenolic standards isorhamnetin, kaempferol, luteolin, apigenin, quercetin, and quercitrin were purchased from Extrasynthese (Genay Cedex, France) and were all standards for UHPLC that is 99\% purity. The identification of individual phenolic compounds in the UHPLC analysis was achieved by comparison of their retention times, UV-Vis spectra and $\mathrm{MS}^{\mathrm{n}}$ spectra data with those of the closest available reference standards and data reported in the literature.

The NMR spectra $\left\{{ }^{1} \mathrm{H},{ }^{13} \mathrm{C}\right.$, DEPT-135, DEPT-90, HSQC, HMBC [71 ms (7 Hz)], COSY $\}$ were measured in $\mathrm{CDCl}_{3}$, on a Bruker Avance $300\left(300.13 \mathrm{MHz}\right.$ for ${ }^{1} \mathrm{H}$ and $75.47 \mathrm{MHz}$ for $\left.{ }^{13} \mathrm{C}\right)$ spectrometer and using TMS as internal standard. Chemical shifts were reported in $\delta$ units (ppm) and coupling constants $(J)$ in $\mathrm{Hz}$. The MS spectra were obtained using $\mathrm{ESI}^{+}$with a Micromass Q-TOF2 mass spectrometer (Manchester, UK). The HRMS-ESI was obtained using a MicroTof spectrometer with Apollo II, using a voltage of $4500 \mathrm{~V}$.

\subsection{Plant Material}

To conduct a representative analysis of the species, several specimens of the whole plant were collected during the flowering period (in August 2015) at "Serra da Estrela" region in Portugal and identified by the botanist Dr. Paulo Silveira, and a voucher specimen was stored in the Herbarium of the Department of Biology, University of Aveiro, Aveiro, Portugal (AVE6611). The plant specimens were dried and divided in roots, stems, and leaves. Each part was ground using a Retsch SKI equipped with a 40-mesh sieve.

\subsection{Extract Preparation}

The pulverized and air-dried parts of G. tridentata were extracted with ethanol using a Soxhlet (three cycles of $48 \mathrm{~h}$ ). The plant part amount was put inside the cartridge and the ethanol was put in the round bottom balloon (10 g plant $/ 250 \mathrm{~mL}$ solvent) and heated during $48 \mathrm{~h}$. The solvent was removed and new ethanol was put in the balloon to make another extraction cycle. This extraction at the ethanol reflux temperature was used to mimic the infusion used in traditional medicine. The ethanol was evaporated under a rotary evaporator, yielding the respective extracts, which were used in the subsequent analyses. Roots extract (R): 83.6 g, 18\% yield; leaves extract (L): $116.5 \mathrm{~g}$, 30\% yield; stems extract (S): $71.9 \mathrm{~g}, 20 \%$ yield.

\subsection{Cell Culture}

The murine macrophage cell line, RAW 264.7, was obtained from the American Type Culture Collection (ATCC: TIB-71) and grown in Dulbecco's modified Eagle's medium (DMEM) supplemented with $10 \%$ fetal bovine serum (FBS), $100 \mathrm{U} / \mathrm{mL}$ of penicillin, and $100 \mu \mathrm{g} / \mathrm{mL}$ of streptomycin. Cells were incubated at $37{ }^{\circ} \mathrm{C}$ in a humidified atmosphere of $95 \%$ of air and $5 \%$ of $\mathrm{CO} 2$ and were used after reaching $70 \%-80 \%$ confluence.

\subsection{Cytotoxicity Assay}

The effect of the different extracts on macrophage viability was assessed by the resazurin assay [48]. Briefly, $4 \times 10^{4}$ cells were plated per well of a 96-well plate, left to stabilize overnight and then incubated with $500,250,100,50$, and $10 \mu \mathrm{g} / \mathrm{mL}$ of each extract during $23 \mathrm{~h}$. The cells were then washed and a medium containing $50 \mu \mathrm{M}$ of resazurin is added. The absorbance was measured at 570 and $600 \mathrm{~nm}$ in a BioTek Synergy HT spectrophotometer (Biotek Instruments, Winooski, VT, USA).

\subsection{The Nitric Oxide Production and NO Scavenging Assays}

Raw 264.7 cells were plated at $3 \times 10^{5}$ cells/well in 48 -well culture plates, allowed to stabilize for $12 \mathrm{~h}$, and then treated with $1 \mathrm{ug} / \mathrm{mL}$ of LPS during $24 \mathrm{~h}$. In certain conditions, cells were pretreated with 
$100 \mu \mathrm{g} / \mathrm{mL}$ of each extract. At the end of the incubation, $100 \mu \mathrm{L}$ of culture supernatants were collected and mixed with an equal volume of Griess reagent [0.1\% $(w / v) N-(1$-naphthyl)ethylenediamine dihydrochloride, and $1 \%(w / v)$ sulfanilamide containing 5\% (w/v) $\mathrm{H}_{3} \mathrm{PO}_{4}$ ] during $30 \mathrm{~min}$, in the dark. After $30 \mathrm{~min}$ incubation in the dark, the absorbance at $550 \mathrm{~nm}$ was measured using a BioTek Synergy HT spectrophotometer. Sodium nitrite $\left(\mathrm{NaNO}_{2}\right)$ solutions $(1-200 \mu \mathrm{M})$ were used to calculate a standard curve. The nitric oxide radical scavenging assay medium containing the NO donor $S$-nitroso- $N$-actyl-D/L-penicillamine (300 $\mu \mathrm{M}$ final concentration) and $100 \mu \mathrm{g} / \mathrm{mL}$ of each extract was incubated at $37^{\circ} \mathrm{C}$ during $3 \mathrm{~h}$. The level of $\mathrm{NO}$ was evaluated through the Griess reaction described above.

\subsection{In Vitro Antioxidant Potential}

Cells were plated at $0.05 \times 10^{6}$ per well in a $\mu$-Chamber slide (IBIDI GmbH, Germany), allowed to stabilize overnight and then treated with $1 \mu \mathrm{g} / \mathrm{mL}$ LPS during $16 \mathrm{~h}$. When indicated, extracts $(100 \mu \mathrm{g} / \mathrm{mL})$ were added $1 \mathrm{~h}$ prior to LPS. At the end of incubation period, cells were washed three times and then loaded with $5 \mu \mathrm{M} \mathrm{H}_{2}$ DCFDA and $0.5 \mu \mathrm{g} \mathrm{mL}{ }^{-1}$ Hoechst in HBSS for 30 min at $37^{\circ} \mathrm{C}$ in the dark. Cells were washed three times with HBSS and analyzed with an Axio Observer Z1 fluorescent microscope (Zeiss Group, Oberkochen, Germany) at 63× magnification.

\subsection{The qPCR Analysis}

Quantitative PCR (qPCR) was used to address the effect of extracts on the LPS-induced transcription of pro-inflammatory genes $I l 1 b$, Nos2, Ptgs2, Il6, and Tnfa. Total RNA was isolated with a TRIzol reagent according to the manufacturer's instructions and the concentration determined by OD260 measurement using a NanoDrop spectrophotometer (Thermo Scientific, Wilmington, DE, USA). After reverse transcription, qPCR reactions were performed in duplicate for each sample on a Bio-Rad CFX Connect. Gene expression changes were analyzed using the built-in CFX Manager software using Hprt1 as a reference gene. This gene was experimentally determined with Genex software (MultiD Analyses AB, Göteberg, Sweden) as the most stable for the treatment conditions used. Primer sequences (Supplementary Table S1) were designed using Beacon Designer software version 7.7 (Premier Biosoft International, Palo Alto, CA, USA) and thoroughly tested.

\subsection{The Western Blot Analysis}

The capacity of G. tridentata extracts to mitigate LPS-triggered expression of the pro inflammatory proteins COX-2 and iNOS was evaluated by Western Blot. After treatments, cells were lysate in a RIPA buffer, protein concentration determined through BCA, and extracts denatured in a Laemmli buffer. Equivalent amounts of protein were applied and separated in SDS-PAGE gel 10\% $(v / v)$, being subsequently transferred to PVDF membranes (Amersham Biosciences, Uppsala, Sweden). The membranes were initially blocked with dry skimmed milk 5\% $(\mathrm{m} / \mathrm{v})$ in Tris-buffered saline containing Tween-20 0.1\% (v/v) (TBS-T), for $1 \mathrm{~h}$ at room temperature. Later, membranes were incubated overnight at $4{ }^{\circ} \mathrm{C}$, with the primary rabbit antibodies to iNOS (1:10,000; R\&D Systems, Minneapolis, MN, USA) and COX-2 (1:10,000; Abcam, Cambridge, UK). After washing in TBS-T, the membranes were incubated at room temperature for $1 \mathrm{~h}$ in secondary alkaline phosphatase-conjugated antibodies. Immunoreactive bands were visualized using ECF substrate on the FLA Thyphoon 9000 imaging system (GE Healthcare). To ensure loading of equal amounts of protein between conditions, membranes were stripped and reprobed with anti-tubulin antibody. The generated signals were analyzed using Total Lab 2009 software (TotalLab Ltd., Durham, NC, USA).

\subsection{UHPLC-DAD-ESI/MS Analysis PCR Analysis}

For the UHPLC-MS analysis, $25 \mathrm{mg}$ of each extract were dissolved in $5 \mathrm{~mL}$ of methanol (final concentration $5 \mathrm{mg} / \mathrm{mL}$ ) and the resulting solutions were filtered through a $0.2 \mu \mathrm{m}$ nylon membrane (Whatman, Oeiras, Portugal). Three independent analyses were carried out for reproducibility. 
This technique was performed using a Thermo Scientific Ultimate 3000RSLC (Dionex) equipped with a Dionex UltiMate 3000 RS diode array detector and coupled to a mass spectrometer. The column used was a Thermo Scientific hypersil gold column $(1000 \times 20 \mathrm{~mm})$ with a part size of $1.9 \mu \mathrm{m}$ and its temperature was maintained at $30^{\circ} \mathrm{C}$. The mobile phase was composed of (A) acetonitrile and (B) $0.1 \%$ formic acid in water $(v / v)$, both degassed and filtered before use. The flow rate was $0.2 \mathrm{~mL} / \mathrm{min}$. The elution gradient was $5 \%$ (solvent B) for $14 \mathrm{~min}, 40 \%$ (solvent B) over $2 \mathrm{~min}, 100 \%$ (solvent B) over $7 \mathrm{~min}$, and the re-equilibration of the column with $5 \%$ of solvent $B$ for $10 \mathrm{~min}$. The injection volume was $2 \mu \mathrm{L}$. UV-Vis spectral data were gathered in a range of 190 to $450 \mathrm{~nm}$ and the chromatographic profiles were documented at 220, 230, 240, and $280 \mathrm{~nm}$. The mass spectrometer used was an LTQ XL linear ion trap 2D equipped with an orthogonal electrospray ion source (ESI). The equipment was operated in a negative-ion mode with electrospray ionization source of $5.00 \mathrm{kV}$ and ESI capillarity temperature of $275^{\circ} \mathrm{C}$. The full scan covered a mass range of 50 to $2000 \mathrm{~m} / \mathrm{z}$. Collision-induced dissociation MS/MS and $\mathrm{MS}^{\mathrm{n}}$ experiments were simultaneously acquired for precursor ions. The detection and quantification limits (LOD and LOQ, respectively) were determined from the parameters of the calibration curves (Table S2).

\subsection{Compounds Isolation and Purification}

The roots extract (R), $53.53 \mathrm{~g}$, was fractionated sequentially, at room temperature, with hexane, dichloromethane, ethyl acetate, acetone, and methanol. Each solvent was added to the extract and stirred for $2 \mathrm{~h}$ to dissolve some compounds, after it was evaporated and purified. From this liquid-solid extraction the hexane fraction $(\mathrm{RH})$ and dichloromethane fraction $(\mathrm{RD})$ were obtained. Therefore, the RH (1.86 g) was separated by column chromatography using a gradient solvent system of dichloromethane:acetone (100:0 to 0:100) and this yielded five subfractions: RH1 (464 mg), RH2 (645 mg), RH3 (160 mg), RH4 (195 mg), and RH5 (147 mg). Lupinifolin 1 (36 mg) was isolated from the subfraction RH5 by TLC and using dichloromethane as eluent. In addition, the RD ( $3.16 \mathrm{~g})$ was separated by column chromatography using a gradient solvent system of dichloromethane:acetone:methanol (100:0:0 to 0:0:100) and this yielded thirty two subfractions which were combined according to their TLC profile. Mundulin $2(5 \mathrm{mg})$ and 3-methoxymundulin $3(3 \mathrm{mg})$ were isolated from the subfraction RD1 by TLC and using hexane:acetone (4:1) as eluent. The compounds NMR data can be found in the Supplementary Information.

\subsection{Statistical Analysis}

All the experiments were repeated three times or more, whenever the replicates were not statistically equal. The results are reported as mean values \pm standard deviation of three independent experiments. Comparisons between two groups were made by the two-sided unpaired Student's $t$ test and multiple group comparisons by One-Way ANOVA analysis, with a Bonferroni's Multiple Comparison or Dunnett's test post-test. Statistical analysis was performed using GraphPad Prism, version 6 (GraphPad Software, San Diego, CA, USA). Significance levels are as follows: ${ }^{*} p<0.05$, ${ }^{* *} p<0.01,{ }^{* * *} p<0.001,{ }^{* * * *} p<0.0001$.

\section{Conclusions}

Overall, the present study identified 20 polyphenolic compounds, among which 18 are flavonoids, in the ethanolic extracts of the roots, stems, and leaves of G. tridentata. Further investigation of fractions from root extracts led to the isolation of three prenylated flavonoids, including a newly described compound identified as 3-methoxymundulin. The biological assays demonstrated strong antioxidant and anti-inflammatory activities, supporting some of the plant traditional use in medicine. A brief analysis of the flavonoid type present in each extract (Table 1; Figure 10), shows that roots and stems are richer in isoflavones, whereas leaves have more flavonols, so it is expected that the anti-inflammatory activity observed is, in leaves due to the flavonols present, and in the other plant parts due to the 
isoflavones. It should be highlighted that both types present several hydroxyl groups in the flavonoid core, a characteristic associated with their anti-inflammatory activity.

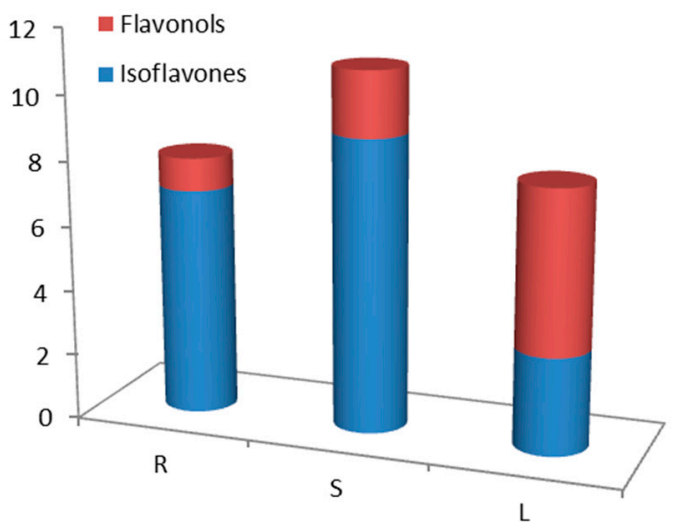

Figure 10. Representative types of flavonoids present in G. tridentata extracts.

Finally, our data indicate the roots of the shrub as an unexplored source of compounds with potential pharmacological and nutraceutical value. Considering that nutraceuticals are functional foods, that not only have nutritional value but also have some medicinal value [49,50], we believe that G. tridentata can be an ingredient of functional foods adding anti-inflammatory properties.

Supplementary Materials: The following are available online: Table S1: Oligonucleotide primer pairs used for qPCR, Lupinifolin 1 NMR data, Mundulin 2 NMR data, 3-Methoxymundulin 3 NMR data.

Author Contributions: Investigation, M.A.M.S.; conceptualization, A.M.S.S.; supervision, A.M.S.S. and B.M.R.N.; validation, D.C.G.A.P. and B.M.R.N.; formal analysis, M.A.M.S., D.C.G.A.P., B.M.R.N., and A.M.S.S.; writing—original draft preparation, D.C.G.A.P.; writing—review and editing, D.C.G.A.P., B.M.R.N., and A.M.S.S. All authors have read and agreed to the published version of the manuscript.

Funding: This research received no external funding.

Acknowledgments: Thanks are due to the University of Aveiro, to the FCT/MEC, and POPH/FSE for the financial support of the QOPNA research unit (FCT UID/QUI/00062/2013) and iBiMED (UID/BIM/04501/2013 and UID/BIM/04501/2019), through national funds and, where applicable, co-financed by the FEDER, within the PT2020 Partnership Agreement and to the Portuguese NMR network (that are partially supported by Infrastructure Project $\mathrm{N}^{\mathrm{o}} 022161$ (co-financed by FEDER through COMPETE 2020, POCI and PORL and FCT through PIDDAC)).

Conflicts of Interest: The authors declare no conflict of interest.

\section{References}

1. The Plant List database. Available online: http://www.theplantlist.org (accessed on 21 January 2020).

2. Talavera, S. Flora Ibérica; CSIC (Centro Superior Investigaciones Científica): Madrid, Spain, 2001.

3. Teixeira, G.; Pereira, A.L. Winged stems in Pterospartum tridentatum: Morphoanatomical study. Acta Bot. Gall. 2004, 151, 103-109. [CrossRef]

4. Novais, M.H.; Santos, I.; Mendes, S.; Pinto-Gomes, C. Studies on pharmaceutical ethnobotany in Arrabida Natural Park (Portugal). J. Ethnopharmacol. 2004, 93, 183-195. [CrossRef] [PubMed]

5. Neves, J.M.; Matos, C.; Moutinho, C.; Queiroz, G.; Gomes, L.R. Ethnopharmacological notes about ancient uses of medicinal plants in Trás-os-Montes (northern of Portugal). J. Ethnopharmacol. 2009, 124, 270-283. [CrossRef] [PubMed]

6. Coelho, M.T.; Gonçalves, J.C.; Alves, V.; Martins, M.M. Antioxidant activity and phenolic content of extracts from different Pterospartum tridentatum populations growing in Portugal. Procedia Food Sci. 2012, 1, 1454-1458. [CrossRef]

7. Gonçalves, S.; Gomes, D.; Costa, P.; Romano, A. The phenolic content and antioxidant activity of infusions from Mediterranean medicinal plants. Ind. Crops Prod. 2013, 43, 465-471. [CrossRef] 
8. Roriz, C.L.; Barros, L.; Carvalho, A.M.; Santos-Buelga, C.; Ferreira, I.C.F.R. Scientific validation of synergistic antioxidant effects in commercialized mixtures of Cymbopogon citratus and Pterospartum tridentatum or Gomphrena globosafor infusions preparation. Food Chem. 2015, 185, 16-24. [CrossRef]

9. Vitor, R.F.; Mota-Filipe, H.; Teixeira, G.; Borges, C.; Rodrigues, A.I.; Teixeira, A.; Paulo, A. Flavonoids of an extract of Pterospartum tridentatum showing endothelial protection against oxidative injury. J. Ethnopharmacol. 2004, 93, 363-370. [CrossRef]

10. Aires, A.; Marrinhas, E.; Carvalho, R.; Dias, C.; Saavedra, M.J. Phytochemical composition and antibacterial activity of hydroalcoholic extracts of Pterospartum tridentatum and Mentha pulegium against Staphylococcus aureus isolates. BioMed Res. Inter. 2016, 5201879. [CrossRef]

11. Martins, V.M.R.L.; Simões, J.; Ferreira, I.; Cruz, M.T.; Domingues, M.R.; Coimbra, M.A. In vitro macrophage nitric oxide production by Pterospartum tridentatum (L.) Willk. inflorescence polysaccharides. Carbohydr. Polym. 2017, 157, 176-184. [CrossRef]

12. Hunter, P. The inflammation theory of disease. EMBO Rep. 2012, 13, 968-970. [CrossRef]

13. Chen, L.; Deng, H.; Cui, H.; Fang, J.; Zuo, Z.; Deng, J.; Li, Y.; Wang, X.; Zhao, L. Inflammatory responses and inflammation-associated diseases in organs. Oncotarget 2018, 9, 7204-7218. [CrossRef]

14. Paulo, A.; Martins, S.; Branco, P.; Dias, T.; Borges, C.; Rodrigues, A.I.; Costa, M.C.; Teixeira, A.; Mota-Filipe, H. The opposing effects of the flavonoids isoquercitrin and sissotrin, isolated from Pterospartum tridentatum, on oral glucose tolerance in rats. Phytother. Res. 2008, 22, 539-543. [CrossRef]

15. Yang, G.; Ham, I.; Choi, H.-Y. Anti-inflammatory effect of prunetin via the suppression of NF- $\kappa B$ pathway. Food Chem. Toxicol. 2013, 58, 124-132. [CrossRef]

16. Byun, E.B.; Sung, N.Y.; Yang, M.S.; Lee, B.S.; Song, D.S.; Park, J.N.; Kim, J.H.; Jang, B.S.; Choi, D.S.; Park, S.H.; et al. Anti-inflammatory effect of gamma-irradiated genistein through inhibition of NF- $\mathrm{BB}$ and MAPK signaling pathway in lipopolysaccharide-induced macrophages. Food Chem. Toxicol. 2014, 74, 255-264. [CrossRef]

17. Hämäläinen, M.; Nieminen, R.; Vuorela, P.; Heinonen, M.; Moilanen, E. Anti-inflammatory effects of flavonoids: genistein, kaempferol, quercetin, and daidzein inhibit STAT-1 and NF- $\mathrm{kB}$ activations, whereas flavone, isorhamnetin, naringenin, and pelargonidin inhibit only NF- $\kappa \mathrm{B}$ activation along with their inhibitory effect on iNOS expression and NO production in activated macrophages. Mediators Inflamm. 2007, 2007, 45673. [CrossRef]

18. Choi, K.-S.; Kundu, J.K.; Chun, K.-S.; Na, H.-K.; Surh, Y.-J. Rutin inhibits UVB radiation-induced expression of COX-2 and iNOS in hairless mouse skin: p38 MAP kinase and JNK as potential targets. Arch. Biochem. Biophys. 2014, 559, 38-45. [CrossRef]

19. Majno, G. Chronic Inflammation. Links with angiogenesis and wound healing. Am. J. Pathol. 1998, 153, 1035-1039. [CrossRef]

20. Roriz, C.L.; Barros, L.; Carvalho, A.M.; Santos-Buelga, C.; Ferreira, I.C.F.R. Pterospartum tridentatum, Gomphrena globosa and Cymbopogon citratus: A phytochemical study focused on antioxidant compounds. Food Res. Inter. 2014, 62, 684-693. [CrossRef]

21. Razali, N.; Razab, R.; Junit, S.M.; Aziz, A.A. Radical scavenging and reducing properties of extracts of cashew shoots (Anacardium occidentale). Food Chem. 2008, 111, 38-44. [CrossRef]

22. Procházková, D.; Boušová, I.; Wilhelmová, N. Antioxidant and prooxidant properties of flavonoids. Fitoterapia 2011, 82, 513-523. [CrossRef]

23. Kole, L.; Giri, B.; Manna, S.K.; Pal, B.; Ghosh, S. Biochanin-A, an isoflavon, showed anti-proliferative and anti-inflammatory activities through the inhibition of iNOS expression, p38-MAPK and ATF-2 phosphorylation and blocking NFkB nuclear translocation. Eur. J. Pharmacol. 2011, 653, 8-15. [CrossRef] [PubMed]

24. Daruházi, Á.E.; Kiss, T.; Vecsernyés, M.; Szente, L.; Szöke, É.; Lemberkovics, É. Investigation of transport of genistein, daidzein and their inclusion complexes prepared with different cyclodextrins on Caco-2 cell line. J. Pharm. Biomed. Anal. 2013, 84, 112-116. [CrossRef] [PubMed]

25. Park, C.E.; Yun, H.; Lee, E.-B.; Min, B.-I.; Bae, H.; Choe, W.; Kang, I.; Kim, S.-S.; Ha, J. The antioxidant effects of genistein are associated with AMP-activated protein kinase activation and PTEN induction in prostate cancer cells. J. Med. Food 2010, 13, 815-820. [CrossRef] 
26. Magiera, S.; Baranowska, I.; Lautenszleger, A. UHPLC-UV method for the determination of flavonoids in dietary supplements and for evaluation of their antioxidant activities. J. Pharm. Biomed. Anal. 2015, 102, 468-475. [CrossRef]

27. Choi, E.J.; Kim, T.; Lee, M.S. Pro-apoptotic effect and cytotoxicity of genistein and genistin in human ovarian cancer SK-OV-3 cells. Life Sci. 2007, 80, 1403-1408. [CrossRef]

28. Baldisserotto, A.; Vertuani, S.; Bino, A.; De Lucia, D.; Lampronti, I.; Milani, R.; Gambari, R.; Manfredini, S. Design, synthesis and biological activity of a novel rutin analogue with improved lipid soluble properties. Bioorg. Med. Chem. 2015, 23, 264-271. [CrossRef]

29. Khan, K.; Pal, S.; Yadav, M.; Maurya, R.; Trivedi, A.K.; Sanyal, S.; Chattopadhyay, N. Prunetin signals via G-protein-coupled receptor, GPR30(GPER1): Stimulation of adenylyl cyclase and cAMP-mediated activation of MAPK signaling induces Runx2 expression in osteoblasts to promote bone regeneration. J. Nutr. Biochem. 2015, 26, 1491-1501. [CrossRef]

30. Choi, E.-Y.; Jin, J.-Y.; Lee, J.-Y.; Choi, J.-I.; Choi, I.S.; Kim, S.-J. Anti-inflammatory effects and the underlying mechanisms of action of daidzein in murine macrophages stimulated with Prevotella intermedia lipopolysaccharide. J. Periodontal Res. 2012, 47, 204-211. [CrossRef]

31. Hershko, D.D.; Robb, B.W.; Luo, G.; Hasselgren, P.-O. Multiple transcription factors regulating the IL-6 gene are activated by cAMP in cultured Caco-2 cells. Am. J. Physiol. Integr. Comp. Physiol. 2002, 283, R1140-R1148. [CrossRef]

32. Falvo, J.V.; Tsytsykova, A.V.; Goldfeld, A.E. Transcriptional control of the TNF gene. Curr. Dir. Autoimmun. 2010, 11, 27-60. [CrossRef]

33. Gonçalves, J.C.; Coelho, M.T.; Diogo, M.G.; Alves, V.D.; Bronze, M.R.; Coimbra, M.A.; Martins, V.M.; Moldão-Martins, M. In vitro shoot cultures of Pterospartum tridentatum as an alternative to wild plants as a source of bioactive compounds. Nat. Prod. Commun. 2018, 13, 439-442. [CrossRef]

34. Kang, J.; Hick, L.A.; Price, W.E. A fragmentation study of isoflavones in negative electrospray ionization by $\mathrm{MS}^{\mathrm{n}}$ ion trap mass spectrometry and triple quadrupole mass spectrometry. Rap. Commun. Mass Spectrom. 2007, 21, 857-868. [CrossRef] [PubMed]

35. Fabre, N.; Rustan, I.; Hoffmann, E.; Quetin-Leclercq, J. Determination of flavone, flavonol, and flavanone aglycones by negative ion liquid chromatography electrospray ion trap mass spectrometry. J. Am. Mass Spectrom. 2001, 12, 707-715. [CrossRef]

36. March, R.E.; Miao, X.-S. A fragmentation study of kaempferol using electrospray quadrupole time-of-flight mass spectrometry at high mass resolution. Int. J. Mass Spectrom. 2004, 231, 157-167. [CrossRef]

37. Mok, S.-Y.; Kim, H.M.; Lee, S. Isolation of astragalin from flowers of Rhododendron mucronulatum for. albiflorum. Hort. Environ. Biotechnol. 2013, 54, 450-455. [CrossRef]

38. Xiao, X.; Xu, L.; Hu, H.; Yang, Y.; Zhang, X.; Peng, Y.; Xiao, P. DPPH Radical scavenging and postprandial hyperglycemia inhibition activities and flavonoid composition analysis of Hawk tea by UPLC-DAD and UPLC-Q/TOF MS ${ }^{\mathrm{E}}$. Molecules 2017, 22, 1622. [CrossRef]

39. Ferreira, F.M.; Dinis, L.T.; Azedo, P.; Galhano, C.I.C.; Simões, A.; Cardoso, S.M.; Domingues, M.R.M.; Pereira, O.R.; Palmeira, C.M.; Peixoto, F.P. Antioxidant capacity and toxicological evaluation of Pterospartum tridentatum flower extracts. CyTA J. Food 2012, 10, 92-102. [CrossRef]

40. Ibrahim, R.M.; El-Halawany, A.M.; Saleh, D.O.; El-Naggar, E.M.B.; El-Shabrawy, A.E.O.; El-Hawary, S.S. HPLC-DAD-MS/MS profiling of phenolics from Securigera securidaca flowers and its anti-hyperglycemic and anti-hyperlipidemic activities. Brazilian J. Pharmacogn. 2015, 25, 134-141. [CrossRef]

41. Kang, J.; Price, W.E.; Ashton, J.; Tapsell, L.C.; Johnson, S. Identification and characterization of phenolic

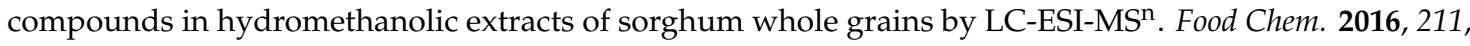
215-226. [CrossRef]

42. Yuzuak, S.; Ballington, J.; Xie, D.-Y. HPLC-qTOF-MS/MS-based profiling of flavan-3-ols and dimeric proanthocyanidins in berries of two muscadine grape hybrids FLH 13-11 and FLH 17-66. Metabolites 2018, 8, 57. [CrossRef]

43. Yusook, K.; Weeranantanapan, O.; Hua, Y.; Kumkrai, P.; Chudapongse, N. Lupinifolin from Derris reticulata possesses bactericidal activity on Staphylococcus aureus by disrupting bacterial cell membrane. J. Nat. Med. 2017, 71, 357-366. [CrossRef] [PubMed] 
44. Alavez-Solano, D.; Reyes-Chilpa, R.; Jiménez-Estrada, M.; Gómez-Garibay, F.; Chavez-Uribe, I.; Sousa-Sánchez, M. Flavanones and 3-hydroxyflavanones from Lonchocarpus oaxacensis. Phytochemistry 2000, 55, 953-957. [CrossRef]

45. Sutthivaiyakit, S.; Thongnak, O.; Lhinhatrakool, T.; Yodchun, O.; Srimark, R.; Dowtaisong, P.; Chuankamnerdkarn, M. Cytotoxic and antimycobacterial prenylated flavonoids from the roots of Eriosema chinense. J. Am. Chem. Soc. 2009, 72, 1092-1096. [CrossRef]

46. Itoigawa, M.; Ito, C.; Ju-Ichi, M.; Nobukuni, T.; Ichiishi, E.; Tokuda, H.; Nishino, H.; Furukawa, H. Cancer chemopreventive activity of flavanones on Epstein-Barr virus activation and two-stage mouse skin carcinogenesis. Cancer Lett. 2002, 176, 25-29. [CrossRef]

47. Reyes-Chilpa, R.; Baggio, C.H.; Alavez-Solano, D.; Estrada-Muñiz, E.; Kauffman, F.C.; Sanchez, R.I.; Mesia-Vela, S. Inhibition of gastric $\mathrm{H}+, \mathrm{K}+$-ATPase activity by flavonoids, coumarins and xanthones isolated from Mexican medicinal plants. J. Ethnopharmacol. 2006, 105, 167-172. [CrossRef]

48. Brien, J.O.; Wilson, I.; Orton, T.; Pognan, F. Investigation of the Alamar Blue (resazurin) fluorescent dye for the assessment of mammalian cell cytotoxicity. Eur. J. Biochem. 2000, 267, 5421-5426. [CrossRef]

49. Nasri, H.; Baradaran, A.; Shirzad, H.; Rafieian-Kopaei, M. New concepts in nutraceuticals as alternative for pharmaceuticals. Int. J. Prev. Med. 2014, 5, 1487-1499.

50. Bull, E.; Rapport, L.; Lockwood, B. What is a nutraceutical? 2000. Available online: https://www. pharmaceutical-journal.com/1-what-is-a-nutraceutical/20002095.article (accessed on 11 February 2020).

Sample Availability: Samples of the compounds are not available from the authors.

(C) 2020 by the authors. Licensee MDPI, Basel, Switzerland. This article is an open access article distributed under the terms and conditions of the Creative Commons Attribution (CC BY) license (http://creativecommons.org/licenses/by/4.0/). 